\title{
Les ressources hydroélectriques de l'Amérique centrale
}

\section{Hydro-electric resources in Central America}

\author{
PAR A. PFAFF \\ INGÉNIEUR DES PONTS ET CHAUSSÉES
}

\begin{abstract}
Au cours de plusieurs missions d'assistance technique échelonnées sur une dizaine d'années, l'auteur a été amené $\dot{a}$ étudivr divers problèmes d'aménagement hydro-électriques en plusieurs pays d'Amérique latine, tantốt pour le compte de l'Assistance technique des Nations-Unies, tanlôt en qualité d'ingénieur-conseil plus spécialement attaché alors d̀ l'étude d'un avantprojet particulier.

Entre 1953 et 1955, it a visité d̀ plusieurs reprises les pays de l'isthme centro-américain. C'est ainsi qu'il a cherché, dans l'article ciaprès, d faire ie point de l'équipement hydroélectrique actuel de l'amérique centrale et des possibilités futures de mise en valenr de ses ressources potentielles.
\end{abstract}

\begin{abstract}
During several technical assistance missions, spread out over a period of some ten years, the author had the task of examining a number of hydro-electric development projects in Latin America. The work was sometimes done for the United Nations technical assistance organization and at other times as consulting engineer. In the latter case the investigations were concerned with particular preliminary projects. Between 1953 and 1955 he visited the countries of the Central America isthmus on several occasions.

It is with this background that he has attempted, in the following article, to sum up the existing hydro-electric developments in Central America, and to point out the resources awaiting to be developed.
\end{abstract}

\section{GENERALITÉS}

L'Amérique centrale est une région généralement assez peu connue, et il n'est sans doute pas tout à fait inutile, avant d'entrer dans le vif du sujet, de rappeler quelques notions ayant trait à sa situation géographique et économique.

Si l'on jette un regard sur la carte (fig. 1), on voit que l'isthme centro-américain, restrictivement appelé chez nous « isthme de Panama», et qui unit entre le Mexique et la Colombie les deux continents du Nouveau Monde, comprend, du $18^{\circ}$ au $7^{\circ}$ parallèle, six pays : Guatemala, Salvador, Honduras, Nicaragua, Costa-Rica et Panama.

La côte Pacifique suit une direction générale nord-ouest - sud-est, parallèle à la chaîne volcanique, tandis que la côte baignée par la mer des Antilles ou Caraïbe présente des troncons nordsud et ouest-est où se développent des plaines généralement plus étendues que du côté du Pacifique.

\section{Géologie et relief}

Au point de vue géologique, l'Amérique centrale est une des régions les plus complexes du monde. Dans sa partie nord-ouest, du Chiapas mexicain jusqu'au nord de la dépression des grands lacs nicaraguayens, les montagnes s'étendent en sierras dirigées d'ouest en est. C'est le système orogénique dit «Antillais », qui constitue le noyau le plus ancien de l'Amérique centrale; ces chaînes montagneuses restées au-dessus du niveau de la mer depuis les premiers temps géologiques se prolongent sous la mer jusqu'aux grandes Antilles (Cuba, Haïti, Jamaïque et Porto Rico). Ce système se distingue de celui plus récent, dit « Andin », formé par un plissement qui, parallèle à la côte Pacifique, renferme le plus grand nombre des volcans.

Le sud-est de l'isthme est une région géologi- 


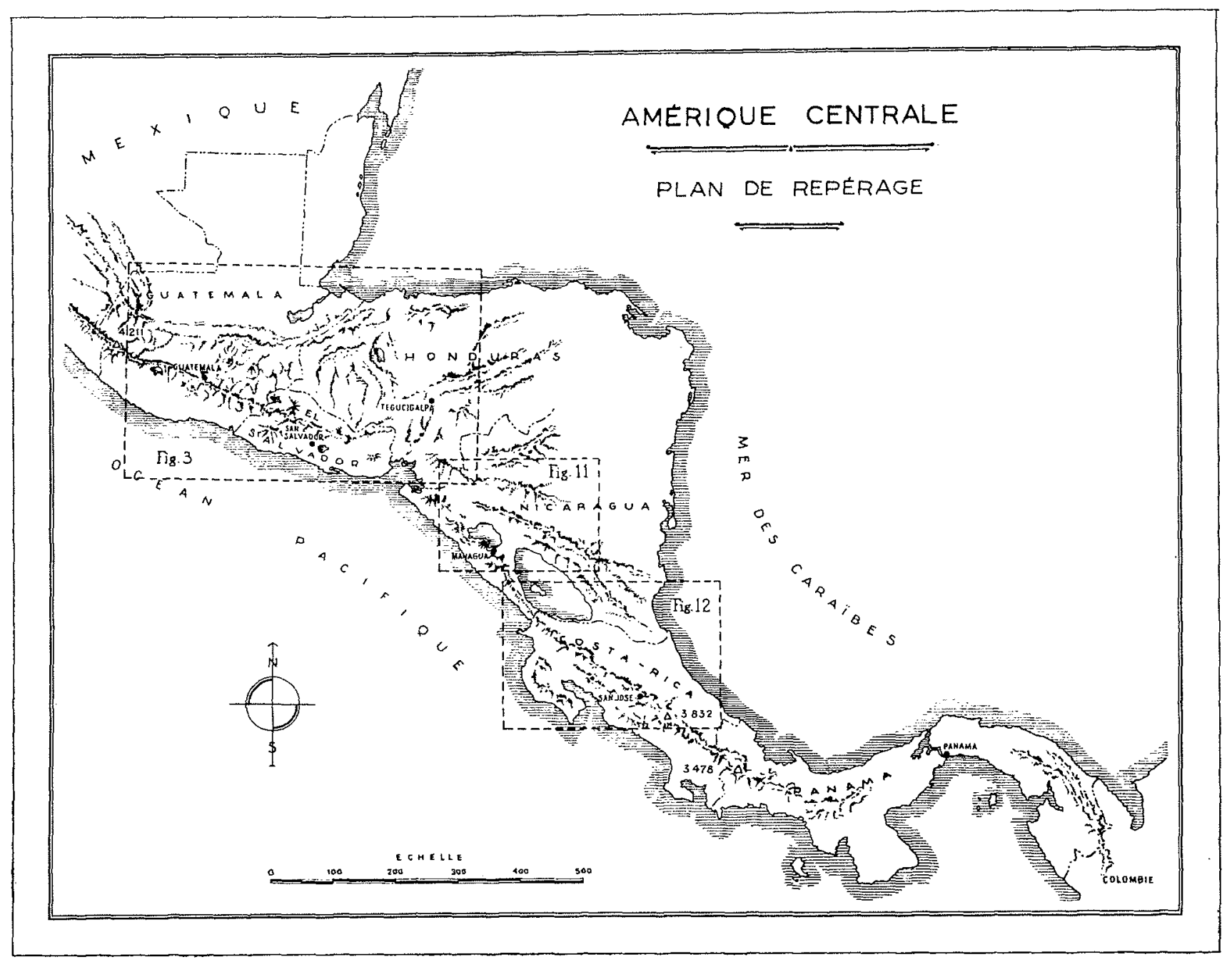

FIG. 1

Carte d'Amérique centrale (plan de repérage).

que distincte, d'origine principalement volcanique, dont la formation considérée comme récente a obstrué à partir du milieu du Miocène et durant le Pliocène une grande ouverture qui unissait jusque-là les deux océans.

Les chaînes les plus anciennes du plissement antillais sont principalement formées de roches cristallines anciennes et sédimentaires. Elles occupent une grande étendue du Honduras et du Guatemala, ot des crêtes de $3.000 \mathrm{~m}$ d'altitude enserrent des vallées profondes et parallèles dont les versants sont coupés de ravins.

Mais la plus grande partie des montagnes du nord-ouest de l'isthme centro-américain date seulement du Pliocène. Parmi ces montagnes non volcaniques, les sommets, qui atteignent en moyenne $3.500 \mathrm{~m}$, sont en général plus élevés que les grands volcans de la chaîne côtière, si lon excepte cependant le volcan Tajumulco, point culminant de $4.211 \mathrm{~m}$.
De grandes dépressions de 1.000 à $1.600 \mathrm{~m}$ d'altitude séparent ces montagnes et donnent passage à des fleuves importants.

Mais la caractéristique la plus remarquable du relief est bien cette suite de pics coniques qui igrene tout au long du Pacificue plus de 100 volcans principaux et 150 plus petits dont un grand nombre sont en activité, et qui sont à l'origine de nombreux tremblements de terre dont certains ont détruit, à plusieurs reprises, des villes entières [Antigua Guatemala (1) en 1770, Managua en 1931].

Une autre caractéristique cst la nature des terrains de surface très souvent formés d'épaisses couches de cendres volcaniques et de laves, facilement entraînées par l'érosion, et qui ont transformé bien des vallées en plaines fertiles et

(1) C'est à la suite de sa destruction que l'ancienne capitale, aujourd'hui appelćc Antigua, a été transférée à l'emplacement de l'actuelle Guatemala. 
peuplées. De nombreux lacs occupent les dépressions créées par l'action volcanique (Atitlan au Guatemala, Guija au Salvador, Nicaragua, etc.).

Les plus grandes plaines se trouvent soit au nord, dans la péninsule de Yucatan qui abrita la civilisation du vieil empire Maya, soit à l'est du Honduras et du Nicaragua sur la côte caraïbe. La plaine côtière du Pacifique, principalement composće d'alluvion volcanique, n'est qu'une longue bande d'une cinquantaine de kilomitres de largeur.

Le sud-est de l'isthme comprend les élats de Costa-Rica et Panama. Nous avons vu qu'il est de formation rolcanique récente. Au Panama, la topographie est très accidentée, bien que dans la majeure partie du pays les collines ne dépassent guère $500 \mathrm{~m}$ d'altitude, sauf à l'ouest où, près de la frontière, les cordillères de Costa Rica se prolongent avec de hauts sommets, parmi lesquels le volcan Chiriqui culmine à $3.478 \mathrm{~m}$.

Au Costa Rica, la chaine forme une épine dorsale qui occupe toute la longueur du pays et renferme des volcans importants et élevés, dont certains sont en activité quasi permanente (volcan Irazù, $3.750 \mathrm{~m}$ ). Toutefois, le sommet culminant $(3.832 \mathrm{~m})$, le Chirripo grande, n'est pas volcanique. La route interaméricaine franchit la cordillère au pied de cette montagne, à plus de $3.300 \mathrm{~m}$ d'altitude.

Ce bref apercu sur le relief de l'Amérique centrale pourrait donner à croire que des sites économiques d'aménagement de hautes chutes doivent se trouver aisément, ce qui serait contraire à la réalité.

En effet, d'une manière générale, les fortes dénivellations se rencontrent surtout sur le versant Pacifique, où les étiages sont longs et sévères. Et ceci nous amène tout naturellement à parler du climat.

\section{Climat}

Le climat, extrêmement varié, est chaud et tropical à basse altitude et se rapproche d'un printemps perpétuel atx environs de $1.500 \mathrm{~m}$ d'altitude. En gros, il existe une saison sèche et une saison pluvieuse bien marquée le long de la côte pacifique, beaucoup moins sur le versant atlantique. Le long de la chaîne volcanique côtière, les zones de plantations de café du Guatemala, qui se situent entre 700 et $1.600 \mathrm{~m}$, recoivent par exemple de $4.500 \mathrm{~mm}$ à $5.500 \mathrm{~mm}$ de précipitations annuelles, durant une saison humide de huit à neuf mois.

La carte ci-jointe donne une division en zones de différentes caractéristiques pluviométriques (fig. 2). II s'agit évidemment d'une représentation assez sommaire mais qui donne une idée de la variété des régimes des cours d'eau.

\section{Economie}

L'économie actuelle de l'Amérique centrale est avant tout agricole. Plus des trois quarts de la population active est occupée à des tâches agricoles et plus de $90 \%$ des exportations des divers pays provient de la terre.

L'agriculture a pour but soit la consommalion locale, soit le commerce. La première, qui existait déjà avant la conquête espagnole, produit principalement le mais, les haricots noirs, la pomme de terre, le riz et les divers aliments nécessaires à l'alimentation locale. Elle se pratique dans de petites exploitations individuelles soit indépendantes soit à l'intérieur des grands domaines. Ce qui n'est pas consommé sur place est vendu au marché roisin.

I’agriculture commerciale est de deux sortes, contrôlée par les intérêts locaux ou par des intérêts étrangers. Dans le premier groupe, le produit principal est le café, quoiqu'il y ait à noter depuis quelque temps une tendance à s'évaler de la monoculture, et à adjoindre à la cullure du café celle de la canne à sucre, du colon, du sésame et de la fibre. Ce genre d'agriculture est l'apanage de fermes importantes, on haciondas, dont les propriétaires sont de riches particuliers autochtones ou étrangers et oì travaillent des Indiens et métis. Au Guatemala, par exemple, $80 \%$ de la production du café provient de 1.500 domaines qui emploient 426.000 ouvriers agricoles. Evidemment, la culture du caf́́, exigeant certaines conditions climatologicues, se trouve concentrée dans les parties moyennes des versants montagneux.

Quant aux plantations exploitées par les capitaux étrangers, il s'agit surtout des bananeraies appartenant aux compagnies américaines, notamment la United Fruit Company.

Cette culture, qui était auparavant à pou pris uniquement localisće sur la còte humide des Caraïbes, a dû être transférée partiellement, par suite de la « maladie de Panama $»$, des régions côtières humides de l'Atlantique à celles plus sèches du Pacifique, au Guatemala el au Costa Rica, où des irrigations sont nécessaires. Les terres abandonnées par les bananiers sont affectées à d'autres cultures : cacao, huile de palme, etc.

Il existe d'autres activités économiques relativement moins considerables mais imporlantes cependant. On peut citer :

- L'élevage, plutôt concentré dans les savanes proches du Pacifique, mais qui se pratique cependant un peu partout;

- I.es mines d'or et d'argent du Fonduras et du Nicaragua;

-. L'industric des bois tropicaux, acajou, bois de Brésil, bois de rose, ébène. 


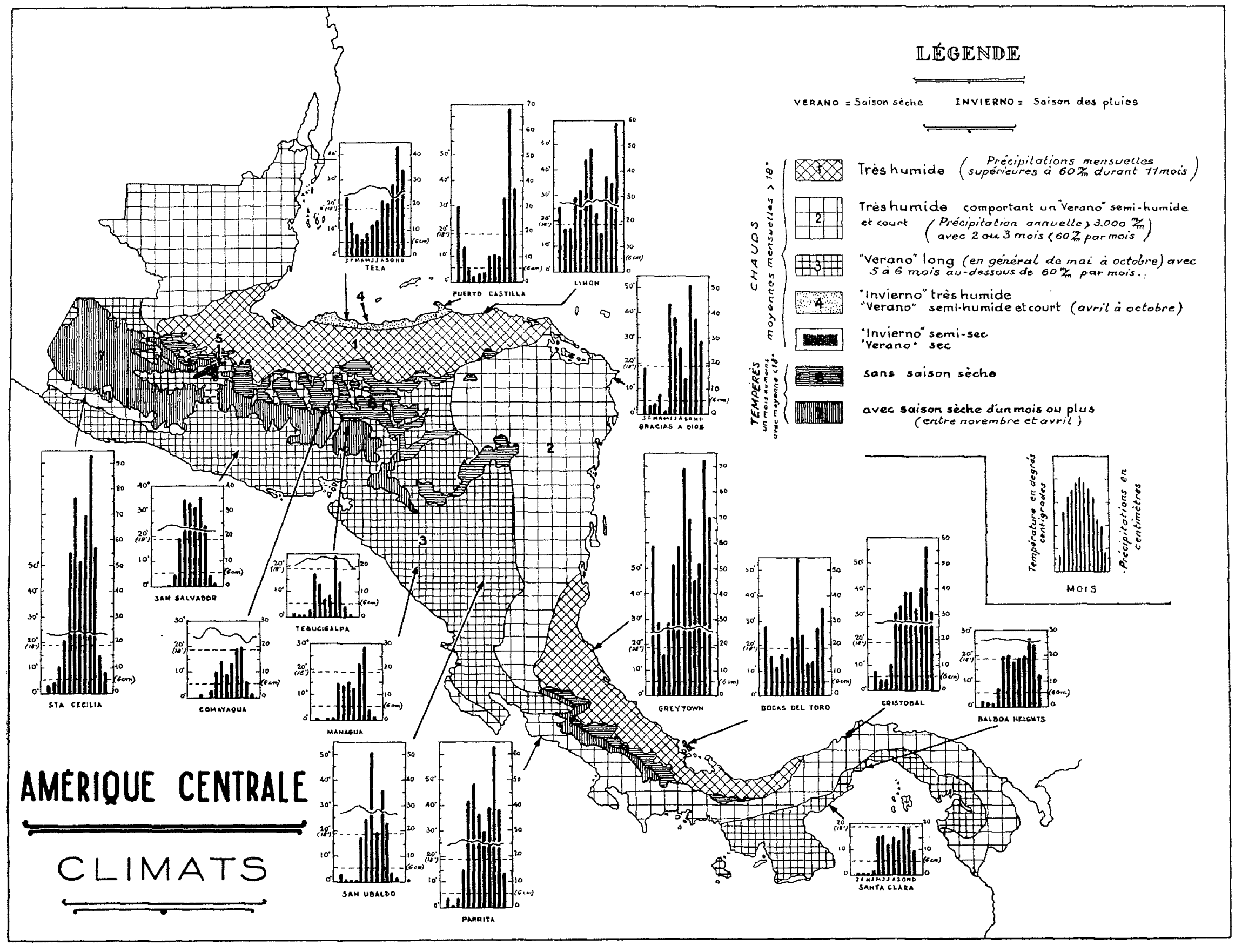


Mais les difficultés d'accès ont laissé à l'état vierge des bois énormes qui restent à exploiter, sauf peut-être au Honduras britannique où cette industrie est la plus développée.

Enfin, la production d'objets manufacturés, quoique encore peu développée, est en net progrès depuis la dernière grande guerre. Limitée seulement par le manque d'énergie électrique, de moyens de transport et de main-d'œuvre qualifiée plus encore que par celui des capitaux, elle se réduit à la fabrication de boissons, chaussures, savon, céramique, ciment, allumettes et cigarettes, pour les besoins locaux.

En ce qui concerne l'énergie électrique, on peut dire que dans la plupart des pays de l'isthme la demande ne peut être satisfaite et s'accrô̂t à une cadence élevée, dépassant par exemple un taux annuel de $15 \%$ pour certains d'entre eux.

D'autre part, les gouvernements intéressés se préoccupent activement, avec l'aide des organismes spécialisés des Nations-Unies, de promouvoir le développement agricole et industriel de leur pay's, ce qui implique une mise en valeur rapide des ressources énergétiques locales, au premier plan desquelles se situe l'énergie hydraulique.

La possibilité de produire de l'énergie en abondance et à un prix abordable représente un des éléments essentiels du développement économique, de telle sorte que celui-ci dépendra, avant tout, des conditions financières d'exécution des projets hydro-électriques.

Certains gouvernements l'ont si bien compris qu'ils sont parvenus dans certaines zones à un haut degré relatif de consommation par le jeu de tarifs extrêmement bas. C'est ainsi qu'au Costa Rica par exemple, $90 \%$ des abonnés utilisent déjà la cuisine électrique et que ce pays est celui d'Amérique centrale où la consommation par habitant est la plus élevée.

Ceci n'a été possible que grâce à la mise en service d'aménagements hydrauliques relativement importants. D'autres ressources hydro-électriques existent et leur mise en valeur sera vraisemblablement effective dans un avenir prochain si les études en cours ou à entreprendre montrent qu'elles sont techniquement utilisables dans des conditions techniques et financières satisfaisantes.

Avant d'examiner la situation, dans chaque pays, de l'utilisation de ses ressources hydroélectriques, nous croyons utile de donner dans le tableau ci-après quelques renseignements statistiques.

\begin{tabular}{|c|c|c|c|c|c|c|}
\hline Pays & $\begin{array}{l}\text { Superficie } \\
\left(\mathrm{km}^{2}\right)\end{array}$ & $\begin{array}{c}\text { Nombre } \\
\text { d'habitants }\end{array}$ & $\begin{array}{l}\text { Puissance } \\
\text { installée } \\
\text { globale } \\
(\mathrm{kW})\end{array}$ & $\begin{array}{l}\% \text { d'origine } \\
\text { hydraulique }\end{array}$ & $\begin{array}{l}\text { Prix } \\
\text { moyen }\left({ }^{1}\right) \\
\text { de vente } \\
\text { du. kWh }\end{array}$ & $\begin{array}{l}\text { Consom- } \\
\text { mation } \\
\text { en kWh } \\
\text { par } \\
\text { habitant (2) }\end{array}$ \\
\hline Guatemala..... & 108.900 & 3.000 .000 & 40.000 & $66 \%$ & 0,036 & 260 \\
\hline El Salvador...... & 20.900 & 1.921 .000 & $50.000(3)$ & $80 \%$ & 0,034 & 300 \\
\hline Honduras.... & 115.200 & 1.500 .000 & 15.000 & $21 \%$ & 0,162 & 100 \\
\hline Nicaragua... & 148.000 & 1.120 .000 & 13.300 & $3 \%$ & 0,044 & 200 \\
\hline Costa Rica........... & 50.900 & 843.000 & 85.000 & $84 \%$ & 0,013 & 500 \\
\hline Panama . . .......... & 74.000 & 845.000 & $\gg$ & & $\gg$ & $\gg$ \\
\hline $\begin{array}{l}\text { (2) Dans la zone de la } \\
\text { (1) En dollars U.S.A. } \\
\text { (3) Non compris les }\end{array}$ & $\begin{array}{l}\text { apitale. } \\
\text { 54). } \\
\text { tallations }\end{array}$ & ées e & $20.000 \mathrm{~kW}$. & & & \\
\hline
\end{tabular}




\section{LES RESSOURCES HYDRO-ÉLECTRIQUES \\ (Equipement actuel et projets)}

Nous allons maintenant exposer la situation, au point de vue des aménagements hydro-électriques réalisés ou réalisables, de chacun des pays de l'isthme centro-américain qu'il nous a été donné d'étudier soit, dans l'ordre géographique : Guatemala, El Salvador, Honduras, Nicaragua et Costa Rica.

Il n'est pas dans notre intention, en procédant ainsi, de limiter notre exposé aux seuls équipements réalisables à l'intérieur de frontières politiques. On verra d'ailleurs que certains d'entrè eux devront être précédés d'ententes entre pays voisins. Mais la concentration relative des populations autour des centres urbains, capitales d'états ou de provinces, nécessitera généralement la produclion de l'énergie à l'intérieur des frontières des pays intéressés, et les échanges internationaux d'énergie ne semblent pas devoir être, à de rares exceptions près, une réalité prochaine.

D'une manière générale, les précipitations annuelles sont élevées dans l'isthme tout entier (fig. 1). Les dénivellations importantes donnent lieu soit à des rivières courtes et à fortes pentes (par exemple région côtière sud du Guatemala), soit à de longs fleuves dont les cours inférieurs

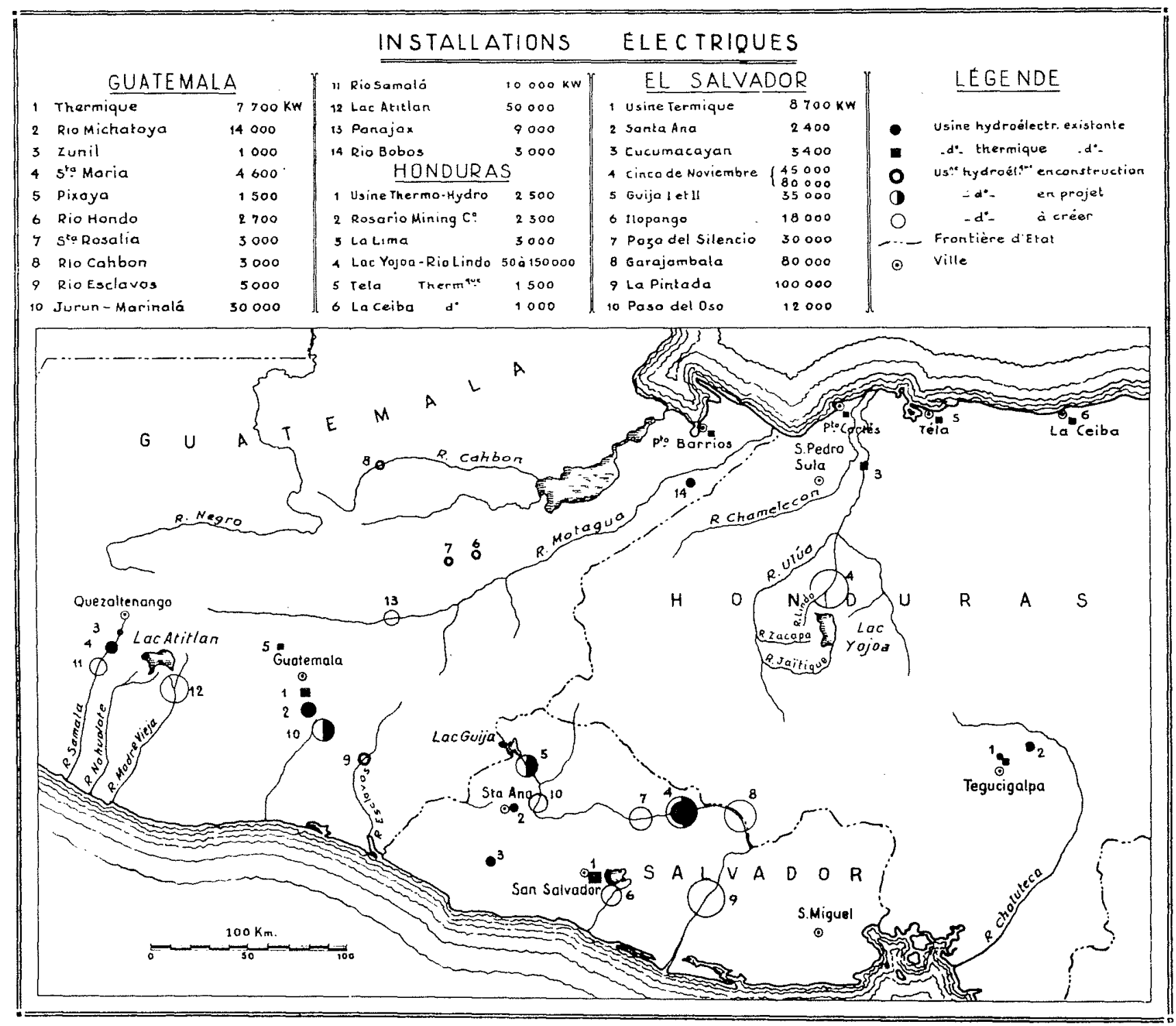

FIG. 3

Région Guatemala. - Honduras. - Salvador.

Installations existantes et ressources potentielles. 


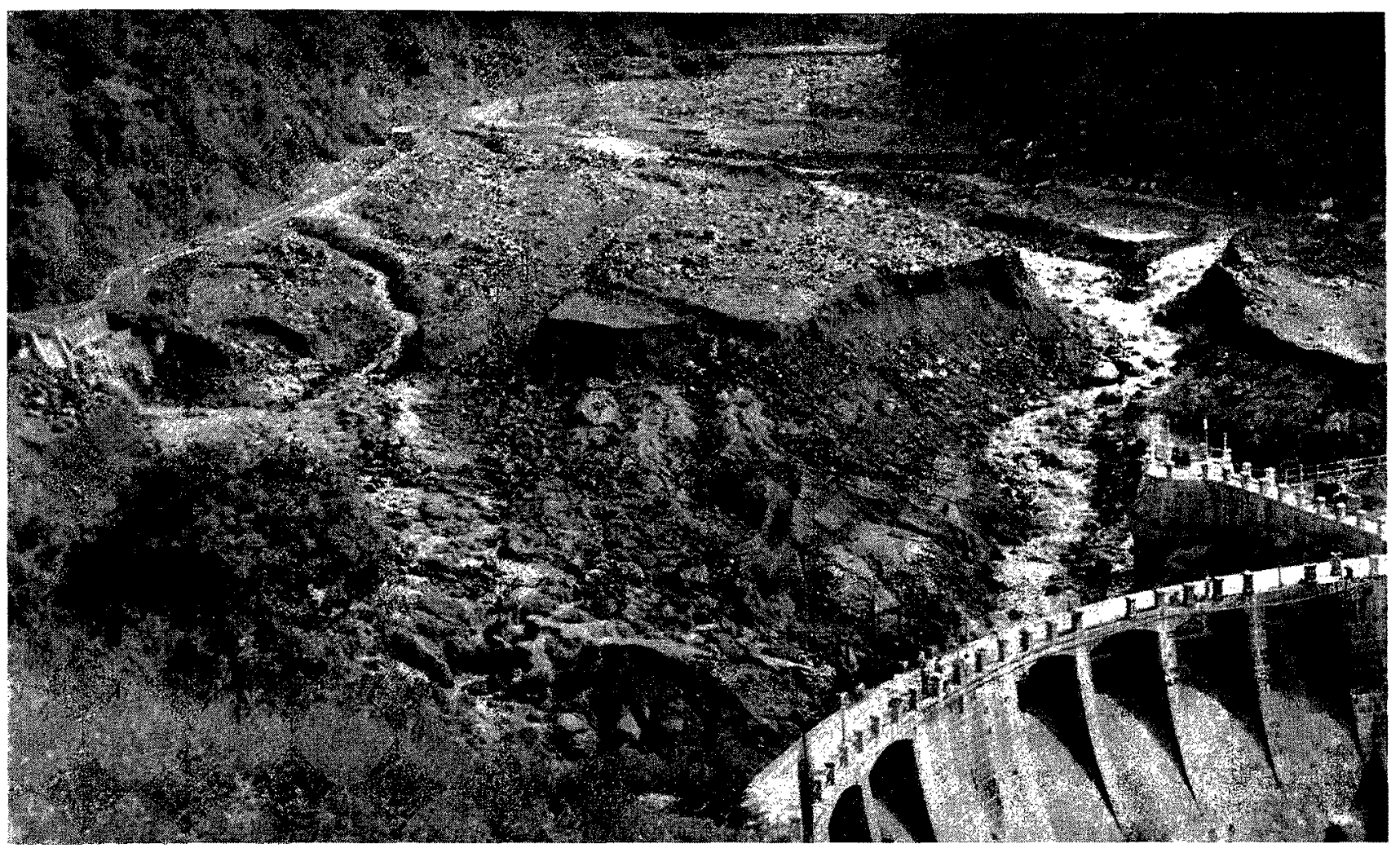

FIg. 4

Barrage sur le rio Samalá comblé par les apports solides.

sont parfois allongés en méandres et nombreux bras morts ne se prêtant à aucun aménagement possible. A peu près toutes les rivières présentent un régime torrentiel marqué, à étiages particulièrement sévères. Traversant des terrains géologiquement récents et composés la plupart du lemps de tufs volcaniques, elles ont des débits solides considérables. Les risques de remblaiement des retenues qui en sont la conséquence (fig. 4), de même que les tremblements de terre fréquents rendent délicats les projets de barrages réservoirs.

Il reste l'utilisation possible de quelques lacs. Mais, à quelques exceptions près, les fuites de leurs cuvettes, qui sont la plupart du temps de formation volcanique, semblent importantes et de longues études s'avèrent indispensables.

Il faut ajouter que malheureusement les stations de jaugeages sont inexistantes sur la plupart des cours d'eau et que l'hydraulicien ne dispose en général d'aucune documentation de base.

\section{Guatemala}

Une étude américaine a évalué à 12.560 GWh (1) annuels le potentiel hydraulique de ce pays.

(1) GWh $=$ millions de $\mathrm{kWh}$.
La politique pratiquée jusqu'à ce jour a été de créer de petites ou moyennes installations au fil de l'eau d'aménagement facile.

En 1954, la puissance hydraulique représentait environ, avec $25.600 \mathrm{~kW}, 66 \%$ de la puissance installée du pays. Mais la totalité de la demande n'était pas satisfaite, et celle-ci s'accroissait très rapidement.

Les seules installations existantes de quelque importance sont celles qui alimentent d'une part la région de la capitale, d'autre part la région de Quezaltenango, deuxième ville $d u$ pays (voir fig. 3).

Les premières utilisent les eaux du rio Michatoya, émissaire naturel du lae Amatitlan qui en régularise le débit. Il s'agit de trois centrales dont les deux premières sont déjà anciennes, établies en cascade sur trois tronçons successifs de la rivière, totalisant une hauteur de chute de $251 \mathrm{~m}$ et une puissance de $12.600 \mathrm{~kW}$. Nous en reparlerons plus loin, ear un projet en cours d'étude met leur existence en jeu.

Les installations qui alimentent la région de Quezaltenango sont des usines de faible puissance au fil de l'eau sur le rio Samalá, existantes ou en construction; leur puissance unitaire est comprise entre 1.000 et $5.000 \mathrm{~kW}$ et peut descendre au-dessous de ces chiffres en périodes d'étiages exceptionnels. Un barrage de $48 \mathrm{~m}$ sur 
le rio Samalá a été comblé par les apports solides (fig. 4).

Il existe en outre quelques projets en cours d'exécution de centrales de 2 à $3.000 \mathrm{~kW}$ en plusieurs points du pays, destinées à satisfaire des consommations locales.

Enfin, la construction d'une centrale au fil de l'eau de $5.000 \mathrm{~kW}$ sur le rio Esclavos a été récemment entreprise. Cependant, la croissance rapide de la demande montre que le problème du manque d'énergie dans la région centrale ne pourra être résolu qu'avec un apport nouveau substantiel.

C'est vers la réalisation de projets hydroelectriques plus importants qu'il y a lieu de se diriger aujourd'hui sans exclure la nécessité de petites installations locales de l'ordre de $500 \mathrm{~kW}$ dont un certain nombre sont d'ailleurs en cours de réalisation.

Parmi ces projets nous décrivons ci-après ceux qui méritent de retenir l'attention.

\section{Aménagement du rio Michatoya. - Projet "Centrale de Marinalá ».}

Le lac Amatitlan, situé à une vingtaine de $\mathrm{km}$ au sud de la capitale, est une belle étendue d'eau de $15 \mathrm{~km}^{2}$ de superficie, entourée de volcans de
$2.500 \mathrm{~m}$ à $4.000 \mathrm{~m}$ d'altitude. Il a déjà recu un aménagement assez complet comportant les usines hydro-élestriques de Palin, San Luis et El Salto (puissance totale $12.600 \mathrm{~kW}$ ), étagées sur le cours de son émissaire le rio Michatoya (voir fig. 5).

Le projet dit de Marinalà consisterait à prendre les eaux à la cote de restitution de la centrale amont (Palin) et les amener dans la vallée parallèle et plus basse du rio Marinalá.

Les études hydrologiques ont montré que le lac Amatitlan dont le bassin versant est, y compris sa propre superficie, de $376 \mathrm{~km}^{2}$, pouvait laisser écouler un débit moyen régularisé de $2,5 \mathrm{~m}^{3} / \mathrm{s}$, qui représente seulement $18,6 \%$ des précipitations. A ce débit s'ajouterait celui du bassin versant intermédiaire entre la sortie du lac et l'usine de Palin; évalué à $0,5 \mathrm{~m}^{3} / \mathrm{s}$ pour un bassin versant de $132 \mathrm{~km}^{2}$.

Un barrage de prise avec dessableur serait construit à $300 \mathrm{~m}$ environ à l'aval de l'usine de Palin. Entre ce barrage et la cheminée d'équilibre, la conduite d'amenée comporterait une partie d'environ $900 \mathrm{~m}$ en galerie revêtue de $4 \mathrm{~m}^{2}$ de section pour un débit maximum de $6 \mathrm{~m}^{3} / \mathrm{s}$ et une conduite en charge de $1,80 \mathrm{~m}$ de diamètre sur 2,650 km. A partir de la cheminée d'équili-

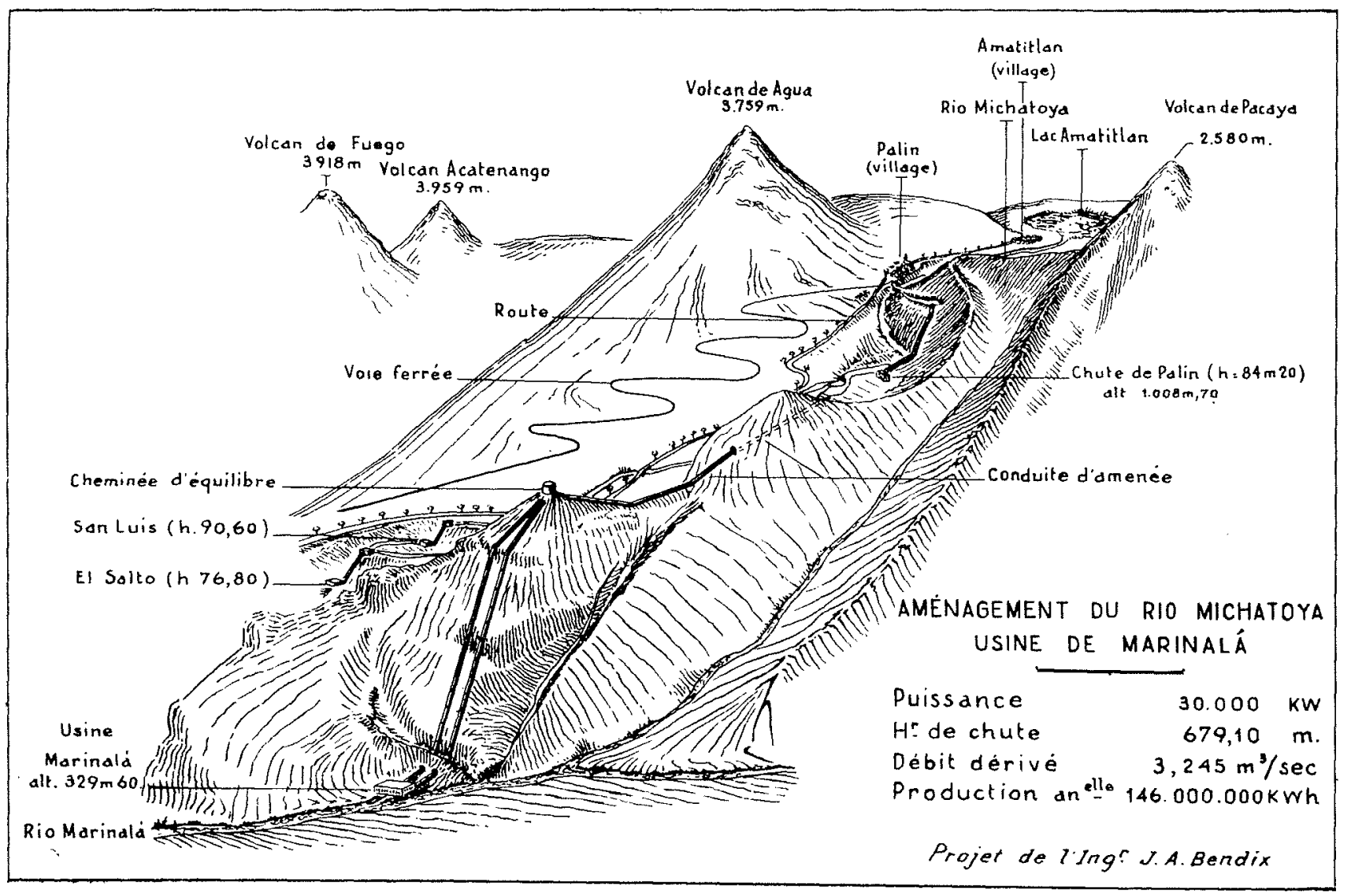

FIG. 5

Aménagement du rio Michatoya et usine de Marinalà. 
bre, deux conduites forcées de $1000 \mathrm{~m}$ de Iongueur amèneraient l'eau à la salle des machines où sont prévus deux groupes Pelton de $15.000 \mathrm{~kW}$.

Ia hauteur de chute nette serait de $640 \mathrm{~m}$ environ.

Afin de disposer d'une régularisation suffisante, il sera nécessaire d'augmenter la tranche utilisable du lac Amatitlan, qui est actuellement de $0,85 \mathrm{~m}$ environ et la porter à $2 \mathrm{~m}$, par abaissement du seuil des vannes de sortie, et dragages dans le lit de l'émissaire. Les indications qui précèdent sont relatives à un avant-projet sommaire, et les études en cours sont susceptibles de modifier quelque peu les ouvrages projetés.

L'inconvénient de ce projet est de réduire dans une grande proportion la productibilité des usines de San Luis et El Salto qui appartiennent à une compagnie privée, la Empresa Electrica de Guatemala.

Cette dernière a opposé un contre-projet qui se confond avec le précédent dans sa partie amont (conduite d'amenée), mais les eaux seraient ramenées vers le rio Michatoya au droit de l'usine de San Luis. La chute nette ainsi obtenue ne serait plus que de $350 \mathrm{~m}$.

L'auteur du projet du rio Marinalá, l'ingćnieur J.-A. BENDIx en estime la rentabilité suffisante, compte tenu des quantités d'énergie à restituer à la Empresa en contrepartie des débits déviés en amont de ses usines. Des études ont été entreprises afin de remplacer, en amont de celles-ci, les eaux détournées par un débit suffisant qui proviendrait des versants situés à une cote supérieure à la restitution de Palin, convenablement draînés. Ces études auraient été concluantes. Nous croyons savoir qu'une décision définitive favorable à l'exécution de ce projet aurait été prise en 1956 .

\section{Le lac Atitlán.}

Bien que le gouvernement du Guatemala ait jusqu'à ce jour cherché à développer seulement les ressources hydro-électriques locales, proches des lieux de consommation, il viendra rapidement un moment où la nécessité de produire des quantités plus considérables d'énergie nécessitera l'aménagement de ressources plus importantes.

Cependant, la quasi-totalité des rivières présente un régime torrentiel très marqué, avec des saisons sèches de longue durée. Le pays est d'autre part entièrement tributaire de l'étranger pour ses importations de charbon ou de pétrole, et son économie ne lui permet pas de les augmenter notablement. Il en résulte la nécessité de chercher à compenser les étiages des rivières par l'utilisation de réserves artificielles ou naturelles.

Les réservoirs artificiels sont de création difficile et de durée précaire en raison du débit solide important. Quant au lac Amatitlan, le plus proche de la capilale, nous arons vu que ses possibilités sont limitées.

Le lac Atitlan, d'une superficie dix fois plus grande avec une étendue de $140 \mathrm{~km}^{2}$, à $70 \mathrm{~km}$ seulement de la capitale et à $25 \mathrm{~km}$ à vol d'oiseau de Queraltenango, admirablement situé d'autre part à $1.600 \mathrm{~m}$ d'altitude et relativement. proche de la plaine côtière, semblait de prime abord devoir apporter une solution idéale au problème. Malheureusement, le lac ne posside aucun émissaire de superficie, et on ne posside sur les variations de son niveau aucune donnée précise s'étendant sur une période de quelque durée.

De même, en ce qui concerne les précipitations, on en est réduit à des extrapolations ou à des hypothèses.

Cependant, sans être particulièrement optimiste sur celles-ci, il semble certain qu'il existe un excédent des apports par rapport aux pertes par évaporation et absorption végétale, qui correspond aux pertes par infiltrations, et qui devrait dépasser $2 \mathrm{~m}^{3} / \mathrm{s}$.

Nous avons pu voir, au cours de prospections faites dans la région sud-est du lac, dont la profondeur maximum dépasse $320 \mathrm{~m}$, des sources puissantes prenant naissance à $450 \mathrm{~m}$ environ en contrebas du niveau des eaux. Ces sources alimentent le rio Madre Vieja et ne connaissent pas d'étiage durant la saison sèche.

Il résulte de ce qui précède qu'il doit être possible de réaliser un certain aménagement hydro-électrique du lac Atitlan. Des études géologiques récemment entreprises devraient montrer si les fuites proviennent seulement des couches supérieures comme il semblerait résulter de certaines investigations antérieures et des hypothèses géologiques jusqu'à présent admises.

S'il en était ainsi, il semble a priori qu'il ne soit pas irréalisable de les obturer, soit par des rideaux d'injections, soit par tout autre procédé dont le choix résultera des études ultérieures.

Il semble d'autre parl qu'il soit possible de dériver vers le lac des eaux provenant de bassins versants voisins, notamment ceux des rios Madre Vieja et Nahualate. La dérivation du haut Madre Vieja nécessiterait une amenée de $14 \mathrm{~km}$ environ, dont $800 \mathrm{~m}$ en galerie.

Une ou plusieurs chutes pourraient alors être aménagées, régularisées par la réserve naturelle du lac. Les usines à créer auraient le caractère d'usines d'accumulation, et devraient être interconnectées d'une part avec les usines au fil de l'eau situées sur des rivières à régime torrentiel, notamment celles des rios Samalá et Esclavos, et d'autre part avec celles du rio Michatoya à déhit régularisé, de manière à produire surtout une énergie de pointe et de saison sèche. Elles devraient donc être fortement suréquipées.

L'aménagement pourrait être effectué soit en 
direction du sud-est vers le rio Madre Vieja, soit au sud-ouest vers le rio Nahualate.

Dans la première hypothèse, cinq usines pourraient être installées :

- L'usine d'Atitlan, qui nécessiterait une prise peu profonde dans le lac, une galerie d'amenée d'environ $2.000 \mathrm{~m}$ et un canal à l'air libre de $3.500 \mathrm{~m}$ suivi d'une conduite en charge de $2.500 \mathrm{~m}$ de long. La hauteur de chute utilisable atteindrait $380 \mathrm{~m}$. La puissance installée pourrait être de l'ordre de $20.000 \mathrm{~kW}$;

- Quatre autres usines en cascade, immédiatement à l'aval de l'usine d'Atitlan, avec les caractéristiques approximatives suivantes:

\begin{tabular}{|c|c|c|c|c|c|}
\hline $\begin{array}{l}\text { Nom } \\
\text { de l'usine }\end{array}$ & 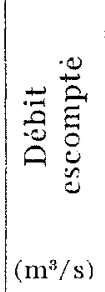 & 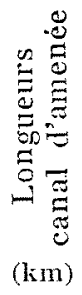 & 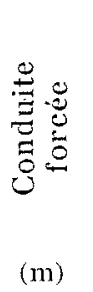 & 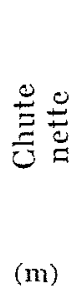 & 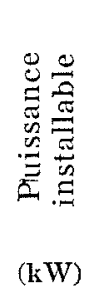 \\
\hline Santa Teresa. & 4,7 & 3,7 & 1.500 & 85 & 7.000 \\
\hline Cacahuate & 5,7 & 0,5 & 1.000 & 115 & 10.000 \\
\hline Ventana. & 7,2 & 2,8 & 800 & 110 & 12.000 \\
\hline Florencia & 8,7 & 2,0 & 500 & 40 & 5.000 \\
\hline Total. & & & & . & 34.000 \\
\hline
\end{tabular}

La puissance installée de l'ensemble des cinq usines pourrait sans doute dépasser $50.000 \mathrm{~kW}$; l'énergie productible a été estimée à environ $240 \mathrm{GWh}$ annuels, sans obturation des fuites, c'est-à-dire avec les seuls débits du rio Madre Vieja dérivé dans le lac, et de ses affluents supérieurs.

Dans la seconde hypothèse, il serait possible d'installer une chute unique de $700 \mathrm{~m}$, ce qui présenterait de grands avantages par rapport à la solution précédente de conception complexe, dont la réalisation conduirait à des difficultés certaines d'exploitation.

On peut remarquer que même sans apports d'eaux dérivées, et sans obturation des fuites, un aménagement pourrait encore être réalisé, de caractère au moins temporaire, mais suffisamment durable pour pouvoir être entrepris dans des conditions économiques satisfaisantes.

Pour un abaissement du niveau moyen du lac d'une trentaine de mètres, un débit moyen supérieur à $2 \mathrm{~m}^{3} / \mathrm{s}$ absolument assuré pourrait en effet être obtenu pendant cinquante ans.

Il est infiniment probable qu'avant d'atteindre une telle dénivellation l'équilibre serait atteint, la diminution des fuites et de l'évaporation devant compenser assez rapidement, selon nous, le débit prélevé.

A ce moment, l'étanchement des fuites deviendrait sans aucun doute plus aisé, et il est vraisemblable qu'on arriverait alors à un aménagement à caractère permanent.

Quoi qu'il en soit, on voit que l'énergie potentielle du lac Atitlan, extrêmement précieuse dans un pays où les rivières ont un étiage long et très bas et où les combustibles d'importation doivent être payés en devises rares, doit pouvoir être utilisée dans un délai relativement proche. Il faut évidemment poursuivre sans désemparer les études topographiques, hydrologiques et géologiques sans les conclusions desquelles aucun projet d'aménagement ne pourra être mené à bonne fin:

\section{Le rio Motagua.}

Nous ne ferons que mentionner ce fleuve tributaire de l'Atlantique. Dans un avenir plus éloigné, il pourrait devenir, comme le rio Lempa au Salvador une source importante d'énergie.

Aucune étude sérieuse n'a encore été entreprise sur cette rivière dont l'aménagement nécessiterail des barrages importants.

Les débits sont caractérisés par des variations saisonnières considérables (de 1 à 600 ), et les retenues à créer risquent d'être rapidement comblées par les apports solides.

$$
\text { *** }
$$

\section{El Salvador}

Avant la mise en service, le 21 juin 1954, du harrage-usine Cinco de Noviembre sur le rio Lempa, qui est actuellement le plus important ensemble hydro-électrique d'Amérique centrale, il n'existait dans ce pays qu'un petit nombre d'usines hydro-électriques dont les deux plus importantes atteignaient $2.300 \mathrm{~kW}$; la puissance installée, d'origine hydraulique, dépassait à peine, avec $10.500 \mathrm{~kW}$, celle des centrales thermiques (fig. 3).

L'augmentation annuelle de la production avait atteint une moyenne de $16 \%$ au cours des trois dernières années dans la région de la capitale, et des restrictions sévères étaient imposées aux consommateurs.

Ces indications sont relatives au secteur public.

Il existe en outre dans le pays des installations privées importantes, les beneficios ou usines de préparation du café, qui peuvent devenir, au fur et à mesure de l'extension du réseau de distribution, des clients du secteur public.

L'aménagement d'une première chute sur le rio Lempa, au lieu dit Chorrera del Guayabo (usine Cinco de Noviembre), a apporté une solution à la crise d'énergie électrique. 
L'étude de ce fleuve a été très sérieusement poursuivie depuis 1942 par Ia Comision Ejecntiva Hidroelectrica del rio Lempa (1) avec l'aide de Harza Engineering Co. Il est maintenant établi que le potentiel hydro-électrique du rio Lempa est suffisant pour pouvoir fournir pendant de longues années la majeure partie de l'énergie dont le pays aura besoin.

Toutefois, le régime torrentiel du fleuve, à peine corrigé par l'aménagement en cours d'achèvement du lac de Guija situé dans la haute vallée d'un de ses principaux affluents, nécessitera un appoint important de centrales thermiques ou d'usines de lac, d'autant plus que la capacité des réservoirs créés par les barrages de retenue ira, selon toute vraisemblance, en diminuant avec le temps. Une solution pourra ĉtre trouvée dans un premier stade, par l'aménagement $d u$ lac Ilopango, et dans un avenir beaucoup plus lointain dans une interconnexion avec le lac Yojoa dont nous parlerons à propos du Honduras.

Nous étudierons successivement :

1. Le potentiel hydro-électrique du rio Lempa;

2. Le barrage-usine « 5 de Noviembre»;

3. L'aménagement du lac de Guija;

4. L'utilisation éventuelle du lac Ilopango.

\section{Le potentiel hydro-électrique du rio Lempa.}

Le rio Lempa est, parmi les tributaires de l'océan Pacifique situćs au sud de Sacramento, en Californie, l'un des plus abondants. II est formé par divers affuents qui ont leur origine dans les montagnes des pays limitrophes (Guatemala et Honduras) et draine un bassin versant de $18.240 \mathrm{~km}^{2}$ à peine inférieur à la surface totale du Salvador $\left(20.900 \mathrm{~km}^{2}\right)$. Une partie seulement de ce bassin versant, soit $58 \%$, se trouve sur le territoire de ce pays.

I.es jaugeages principaux ont été faits à $43 \mathrm{l} / \mathrm{m}$ seulement de son embouchure, en un lieu dit «La Pintada », où un important aménagement est envisagé. Le bassin versant $y$ est de $17.254 \mathrm{~km}^{2}$. L Les faibles débits correspondant à la saison sìche ont une durée de six mois avec un déhit minimum d'étiage de l'ordre de $35 \mathrm{~m}^{3} / \mathrm{s}$, alors que la crue maximum connue aurait dépassé $25.000 \mathrm{~m}^{3} / \mathrm{s}$. Le débit moyen annuel (module) est de $370 \mathrm{~m}^{3} / \mathrm{s}$.

La régularisation même partielle de ces débits entraînerait la création de réservoirs importants.

Nous avons mentionné plus haut qu'il existe une certaine possibilité de régularisation que permet la présence sur un affluent du cours supérieur, du lac de Guïja, dont le bassin ver-

\footnotetext{
(1) La Comision Ejecutiva Hidroelectrica del rio Lempa est un organisme national autonome charge de la mise en valeur du potentiel énersétique du rio lempa et de ses affluents.
}

sant $\left(2.950 \mathrm{~km}^{2}\right)$ représente $17 \%$ de celui du flenve à La Pintada. Nous y reviendrons plus loin.

Fn dehors de l'aménagement actuellement réalisé sur le rio Lempa et de celui projeté pour l'utilisation des eaux du lac de Guija, desquels la productibilité totale atteindra 400 GWh lorsqu'ils auront atteint leur plein développement, d'autres sites ont été retenus pour l'étude d'avant-projets ultérieurs de barrages-usines :

- A $25 \mathrm{~km}$ environ à l'amont du barrage « 5 de Noviembre », le sile de Poza del Silencio qui, avec une chute d'environ $30 \mathrm{~m}$ et compte tenu de la régularisation procurée par le lac de Guijja aménagé, pourrait produire annuellement 132 GWh;

- Sur le haut cours du fleuve, immédiatement à l'aval du confluent de l'émissaire du lac de Guija, aux lieux dits Paso del Oso et El Sunza. Production escomptée 50 à $70 \mathrm{GWh}$;

--. En s'éloignant vers l'aval de l'aménagement « 5 de Noviembre », et sans aucune perte de chute intermédiaire, on rencontrerail successivement les barrages-usines de :

- Guarajambala (hauteur de chute $54 \mathrm{~m}$, production $370 \mathrm{GWh}$ ) qui, novant des terres appartenant au Honduras et s'appuyant sur la rive gauche du fleuve sur le territoire de ce pays, nécessiterait un accord international préalable,

-.. La Pintada (chute $35 \mathrm{~m}$, production 438 GWh.)

Bien entendu, les ćtudes à venir peuvent montrer la nécessité de modifier l'aménagement projeté, mais les indications précédentes montrent que le fleuve Lempa doit pouvoir fournir un jour une énergie annuelle de plus de $1.400 \mathrm{GW}^{\mathrm{h}} \mathrm{h}$.

Cette énergie pourrail même être notablement dépasséc si une interconnexion avec une importante usine de lac se révélait un jour possible, permettant un certain surcquipement des usines en vue de l'utilisation d'une partie des débits sauvages de la saison humide. Nous pensons en particulier au lac Yojoa, au Honduras, pays avec lequel d'intéressants échanges d'énergie pourraient avoir ainsi licu. Mais cela suppose un degró de développement que ce dernier pays surtout est encore loin d'avoir atteint.

\section{Le barrage-usine « 5 de Noviembre \%.}

Cet ensemble a été construit au lieu dit Chorrera del Guayabo, à $60 \mathrm{~km}$ au nord-est de la capitale, et c'est sous ce nom qu'il était désigné jusqu'au jour de son inauguration en juin 1954 (fig. 6) (1).

(1) Les photographies (fig. 6, 7, 9, 10) sont dues a l'obligeance de $M$. V. DE Sora, président de la Comision Ejecutiva del rio Lempa et de l'ingenieur E. L.ma, directeur. 


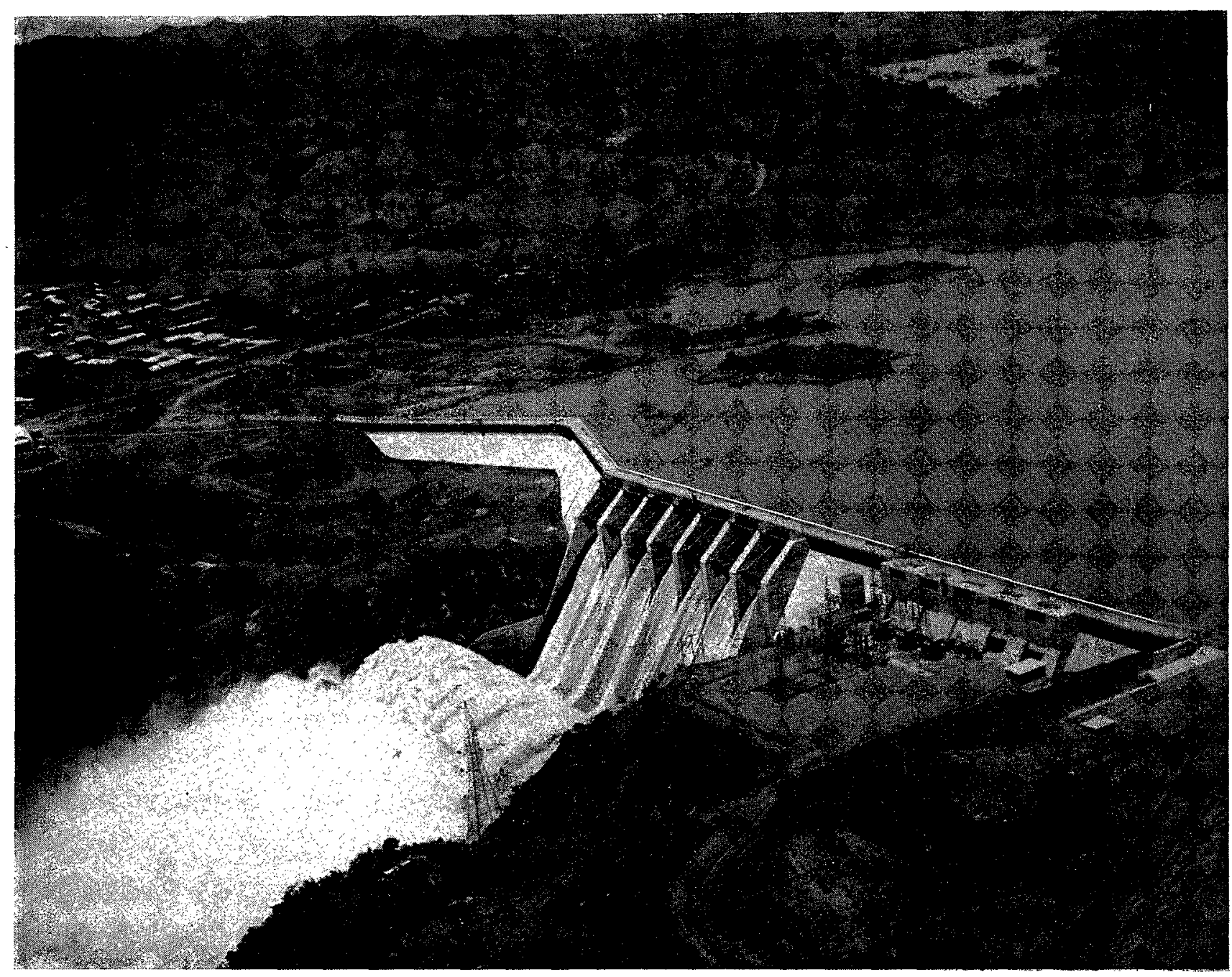

FIG. 6

Barrage-usine «Cinco de Noviembre» sur le rio Lempa (Chorrera del Guayabo). Vue générale des ouvrages.

Le plan d'eau est relevé de $53 \mathrm{~m}$ par un barrage-poids en béton de $450 \mathrm{~m}$ de longueur totale. La partie centrale du barrage, qui atteint une hauteur de $60 \mathrm{~m}$ all-dessus des fondations, est aménagée en déversoir comportant 7 passes fermées de vannes secteur de $12,50 \mathrm{~m}$ de long et $12 \mathrm{~m}$ de hauteur, capables d'évacuer une crue de $18.500 \mathrm{~m}^{3} / \mathrm{s}$ (bassin versant $9.785 \mathrm{~km}^{2}$, plus forte crue connue $15.000 \mathrm{~m}^{3} / \mathrm{s}$ en 1934 ).

Une digue en terre de $11 \mathrm{~m}$ de hauteur et de $250 \mathrm{~m}$ de long ferme une dépression située à $900 \mathrm{~m}$ en amont du barrage. Elle est destinée à être détruite par déversement en cas de crue exceptionnelle, afin d'en assurer l'évacuation avec un dommage restreint.

L'usine (fig. 7) est souterraine, située sur la rive gauche dans le prolongement du barrage; elle a été prévue pour comprendre, dans son stade de développement définitif, cinq groupes de $15.000 \mathrm{~kW}$, dont les deux premiers ont été immédiatement installés et un troisième commandé pour être mis en service en 1956. Les deux derniers groupes sont prévus pour être installés postérieurement à la réalisation tout au moins partielle de l'aménasgement du lac de Guïja (voir ci-dessous).

Chaque turbine Francis à axe horizontal est alimentée directement par un puits vertical. Deux galeries de fuites sont prévues, la premic̀re qui recueille les eaux turbinées par les trois premiers groupes étant seule actuellement construite.

On accède à l'usine par un tunnel d'accès en pente douce.

Chaque groupe est relié à un bloc transformateur oì la tension est ćlevée de 13.800 à $115.000 \mathrm{~V}$.

La retenue est d'environ 400 millions de $\mathrm{m}^{3}$ avec une tranche utilisable théorique de $200 \mathrm{mil}$ lions de $\mathrm{m}^{3}$. 


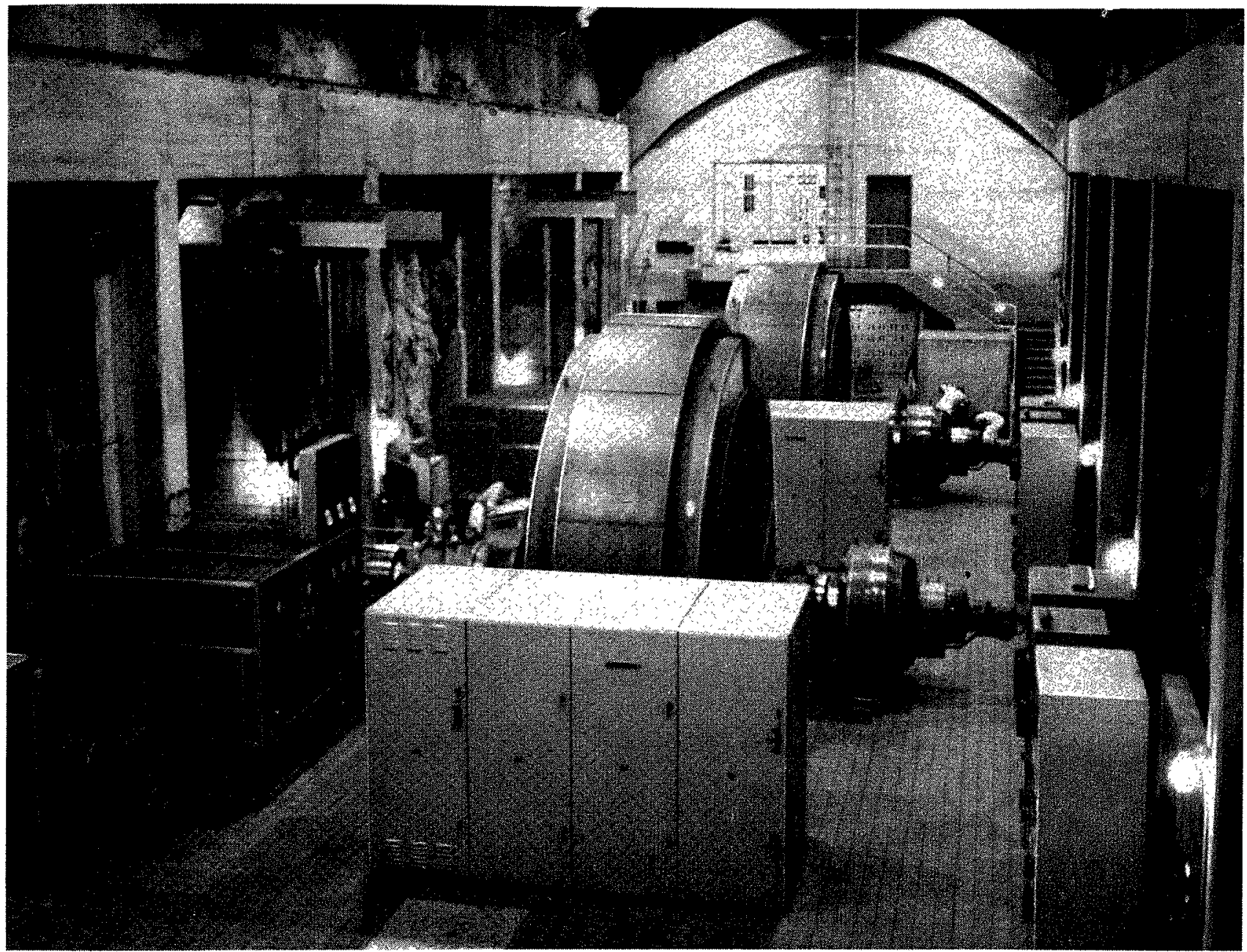

FiG. 7

Vue partielle de l'usine souterraine « Cinco de Noviembre».

\section{L'aménagement du lac de Guïja.}

Le lac de Guïja, situé dans le haut bassin du Lempa, est limitrophe du Guatemala qui supporte la quasi-tolalité de son bassin versant de $2.560 \mathrm{~km}^{2}$ environ.

La superficie proprement dite du lac, soit $45 \mathrm{~km}^{2}$, sa profondeur variable de 20 à $25 \mathrm{~m}$, ainsi que sa situation en tête du lleuve, le destinaient à devenir un régulateur accumulateur d'énergie de saison sèche.

Les études faites par nous-même en 1954, avec l'aide de M. LinnikofF, ingénieur géologue, ont abouti à la conclusion qu'il existail en outre des possibilités d'utilisation très intéressantes de la chute de $75 \mathrm{~m}$ existant sur l'émissaire du lac, le rio Desagüe, affluent du rio Lempa distant de 7 à $8 \mathrm{~km}$.

Actuellement, un barrage (fig. 9 et 10) s'élevant à $6 \mathrm{~m}$ au-dessus du niveau du seuil naturel du lac a été construit sur le rio Desagüe à environ $2 \mathrm{~km}$ à l'aval de ce dernier, et le fond du lit de la rivière a été excavé à l'amont du barrage de manière à laisser une [ranche utilisable de $16 \mathrm{~m}$.

Selon nos études, il serail désirable, pour une utilisation optimum, de surélever encore le barrage de près de $4 \mathrm{~m}$, mais il semble que des considérations étrangères à la technique ne le permettent pas, les terres inondées étant pour la plus grande partie situces en territoire guatemaltèque.

Quoi qu'il en soit, l'aménagement tel qu'il est projeté actuellement comprendrait, outre le barrage sur le rio Desagüe qui devrait ètre prolongé d'un important barrage souterrain destiné à obturer d'importantes fuites sur la rive gauche, deux usines hydro-électriques en cascade :

- Guïja I, de $43 \mathrm{~m}$ de chute nette moyenne, alimentée directement par une prise d'eau adjacente au barrage el une galerie d'amenée de $5,20 \mathrm{~m}$ de diamètre pour un débil maximum de $75 \mathrm{~m}^{3} / \mathrm{s}$, percée sur $300 \mathrm{~m}$ de 


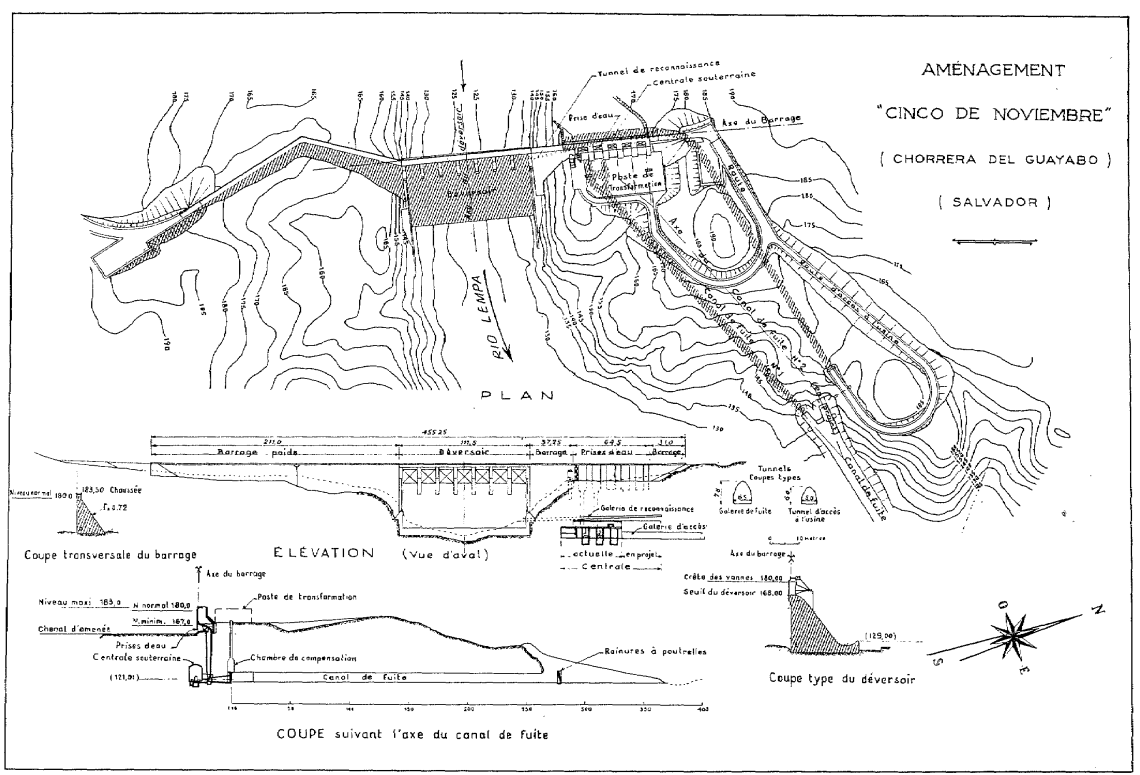




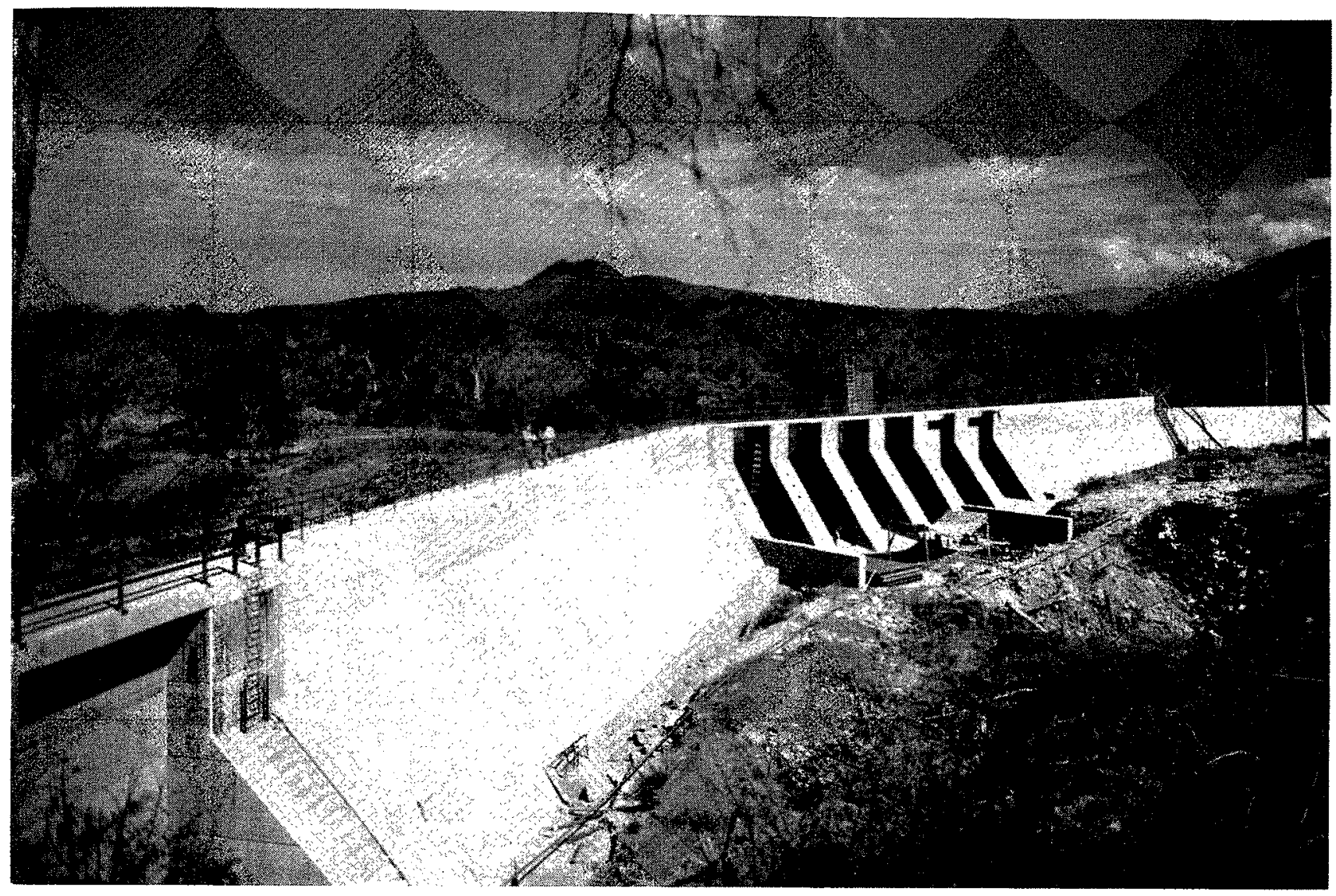

FrG. 9

Barrage de contrôle du lac de Guïja sur le rio Desagüe : aile rive gauche.

longueur à travers un massif de brèche andésitique. Cette usine serait équipée de deux groupes de $12.500 \mathrm{~kW}$.

- Guija II, comprenant un barrage de prise sur le rio Desagüe, immédiatement à l'aval de la restitution de Guijja I, créant une réserve journalière, et une usine de $28 \mathrm{~m}$ de chute nette alimentée par un canal d'amenée à l'air libre de $2.400 \mathrm{~m}$ de longueur et une conduite forcée très courte. La puissance installée à Guïja II serait de $15.000 \mathrm{~kW}$ en un seul groupe.

L'ensemble des deux usines permettrait, sans surélévation du barrage du rio Desagüe, de porter à $400 \mathrm{GWh}$ la productibilité annuelle y compris celle de l'usine « 5 de Noviembre » qui, seule, ne dépasserait guère $200 \mathrm{GWh}$ en année moyenne, en supposant le barrage en service jouant un simple rôle d'accumulation, sans utilisation de la chute du rio Desagüe.

L'usine « 5 de Noviembre » avec ses 5 groupes ne scra pleinement valorisée selon nous qu'après surélévation de la crête du barrage du Desagüe. L'ensemble pourra alors dépasser $500 \mathrm{GWh}$ de productibilité annuelle.

\section{L'utilisation éventuelle du lac Ilopango.}

Nous avons insisté plus haut sur la valeur exceptionnelle de l'énergie de lac, en Amérique centrale, en raison des sécheresses prolongées des rivières, notamment celles du versant pacifique, et de la nécessité d'importer les combustibles. Le Salvador ne fait pas exception à cette règle, et l'utilisation du lac Ilopango présenterait un intérêt certain. Elle n'a malheureusement fait l'objet d'aucune étude approfondie. Ce lac est situé au centre du pays, à une dizaine de kilomètres seulement à l'est de la capitale San Salvador. Il a une superficie de $68,5 \mathrm{~km}^{2}$. Les eaux excédentaires s'écoulent vers le rio Jiboa par un émissaire qui atteint ce fleuve côtier à $6 \mathrm{~km}$ environ du lac (fig. 3).

Des mesures faites il y a une vingtaine d'années lui attribuent un débit moyen de $4,6 \mathrm{~m}^{3} / \mathrm{s}$.

La cuvette du lac est située dans un fossé d'effondrement orienté est-ouest qui fait partie d'une dislocation parallèle à la côte s'étendant sur plusieurs centaines de kilomètres. Elle se trouve ainsi dans une zone instable affectée de mouvements tectoniques dont certains sont extrêmement récents, suivant l'étude faite en 1954 par N. LINNIKOFF, 


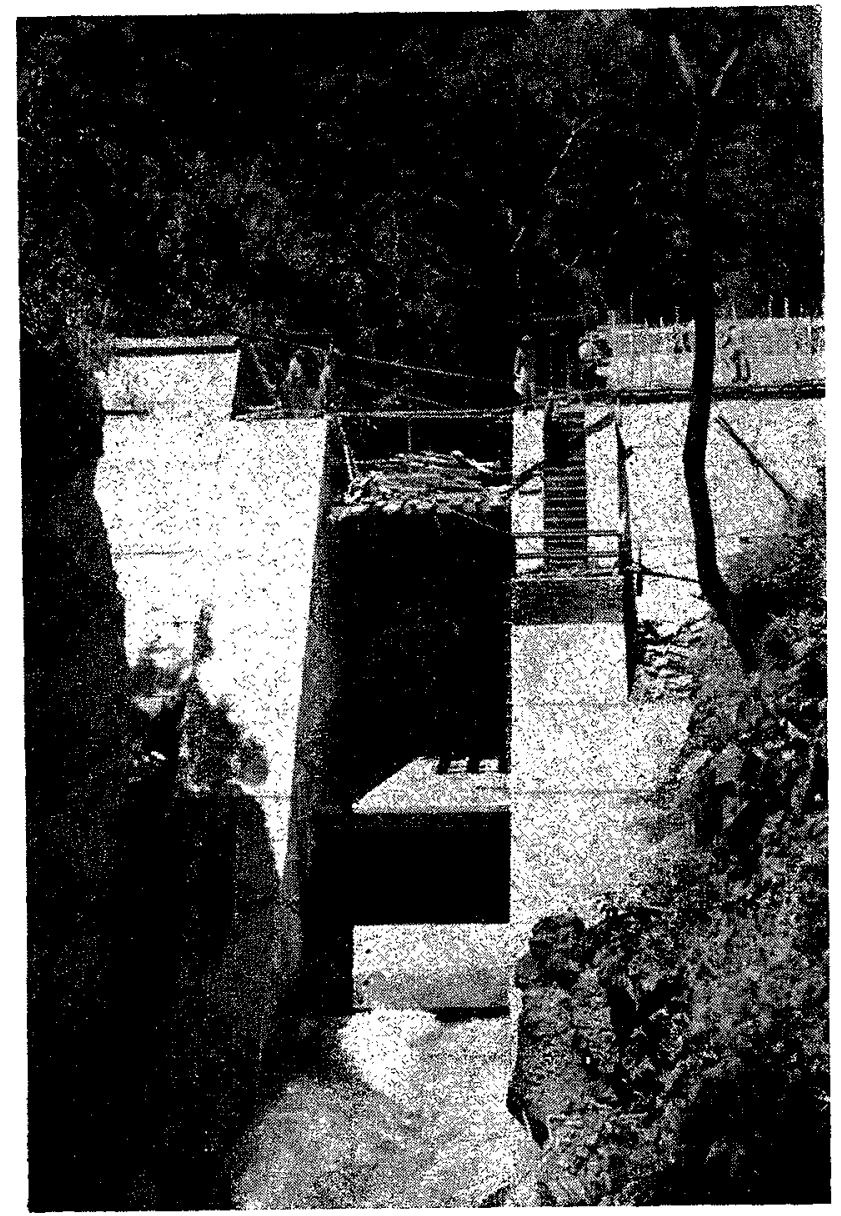

FIG. 10

Barrage de contrôle du lac de Gnïja : partie centrale.

Le niveau $d u$ lac pourrait être contrôlé par un barrage qui, en raison des conditions géologiques, serait construit en terre à la sortie même du lac. Il ne présenterait aucune difficulté particulière de fondation. Entre le niveau du lac à la cote 440 et le rio Jiboa, dont le cours est parallèle à la rive sud-est du lac, une différence de niveau d'environ $220 \mathrm{~m}$ pourrait être utilisée.

La difficulté de l'aménagement réside surtout dans le percement de la galerie d'amenée, non pas tant par suite des terrains traversés (brèche, andésite, poudingue et peut-être basalte), malgré la fréquence des contacts de roches différentes, que par suite des venues d'eau probables, et surtout de l'action non encore éteinte des forces épirogéniques.

Ces inconvénients ne paraissent pas majeurs toutefois. La zone amont de la galerie pourrait même se situer au-dessus du niveau du lac si une étude géologique plus poussée en montrait Ia nécessité, la prise d'eau nécessitant dans ce cas une station de pompage. Il semble, d'autre part, que le tracé de la galerie, qui est en dehors de la zone principale de dislocation, permette un certain optimisme au regard des ruptures éventuelles du souterrain.

L'aménagement du lac donnerait au minimum une énergie annuelle de $75 \mathrm{GWh}$ qui serait réservée pour les pointes et la saison sèche.

$$
*
$$

\section{Honduras}

La zone côtière nord, qui possède avec les vastes plantations de la United Fruit $C^{\circ}$ les villes les plus importantes, et d'autre part la région. de 'Tegucipalpa, capitale administrative, connaissent seules un degré de développement notable (fig. 3).

Au total, la puissance électrique installée dans le pays est encore très faible et ne dépasse guère $15.000 \mathrm{~kW}$, dont $20 \%$ environ seulement d'origine hydraulique. Bien que les tarifs de vente très élevés freinent la consommation, la demande s'accroît cependant d'environ $15 \%$ annuellement.

Par ailleurs, l'importance relative de la production d'origine thermique a amené à rechercher la possibilité de substituer aux centrales Diesel, à plus ou moins brève échéance, des usines hydrauliques.

Il existe au Honduras de nombreuses rivières qui représentent un potentiel hydro-électrique considérable. On peut citer comme l'une des possibilités les plus spectaculaires les cataractes du rio Patuca dans un canyon dénommé Portal del Infierno, mais les aménagements pouvant actuellentent être envisagés conme rentables à brève échéance sont encore peu nombreux, soit que les sites soient trop éloignés des lieux de consommation, soit qu'ils nécessitent des ouvrages relativement trop importants.

L'un d'eux cependant nous parait réunir des conditions extrêmement favorables. Il s'agit du projet d'aménagement du rio Lindo, qui est en fait liè à celui de l'utilisation des eaux du lac Yojoa qui alimente d'ailleurs en partie cette rivière.

\section{Le projet d'aménagement du rio Lindo (lac Yojoa).}

I e lac Yojoa est situé à $115 \mathrm{~km}$ environ au nord-ouest de Tegucigalpa et à $65 \mathrm{~km}$ de San Pedro Sula, ville la plus peuplée du pays, dans la zone côtière nord.

Il est entouré à l'est et à l'ouest de hautes montagnes atteignant $2.740 \mathrm{~m}$ et fermé au nord par une crête qui s'élève seulement jusqu'à $7 \mathrm{~m}$ audessus de son niveau moyen. Il existe au sud un émissaire saisonnier, le rio Jaitique.

Le plan d'eau du lac situé à $640 \mathrm{~m}$ au-dessus du niveau de la mcr a une superficie de $86 \mathrm{~km}^{2}$. Le bassin versant, de $340 \mathrm{~km}^{2}$, recoit des préci- 
pitations abondantes de l'ordre de $2.500 \mathrm{~mm}$ par an.

Le lac perd ses eaux par des fuites dont les résurgences à l'ouest, à peu près constantes et de l'ordre de $12 \mathrm{~m}^{3} / \mathrm{s}$, forment le rio Zacapa. Il semble résulter des premières investigations que ces fuites pourraient être obstruées sans difficultés majeures.

Le rio Lindo qui s'écoule vers le nord serait également formé partiellement par des eaux provenant de fuites du lac.

D’après des mesures pluviométriques et évaporimétriques, le débit moyen régularisé qui pourrait être utilisé après obturation des fuites du rio Zacapa et de l'émissaire sud scrait de l'ordre de $22 \mathrm{~m}^{3} / \mathrm{s}$. En outre, ce débit pourrait être considérablement augmenté par dérivation d'eaux additionnelles à partir des bassins versants de la région située au sud du lac.

I.e rio Lindo, sur lequel s'échelonneraient les aménagements, prend sa source dans les hautes montagnes de Santa Barbará qui longent la rive ouest du lac et se prolongent ensuite vers le nord (fig. 3 ).

Il contourne l'extrémité nord du lac et descend directement vers le nord peu après avoir traversé, au village de rio Lindo, à une centaine de mètres d'altitude, la route de Tegucigalpa à San Pedro Sula qui constitue l'une des principales artères transversales de l'isthme.

Au débit du propre bassin versant de la rivière viennent s'ajouter des eaux souterraines en provenance du lac Yojoa qui lui procurent ainsi une certaine régularisation naturelle.

La pente rapide du rio Lindo qui, à $16 \mathrm{~km} \mathrm{du}$ lac seulement, à la traversée de la route, se trouve déjà à près de $600 \mathrm{~m}$ en contrebas de son niveau moyen, offre de magnifiques possibilités d'aménagements de chutes.

Des avant-projets ont été étudiés qui ne diffèrent qu'en raison de la productibilité demandée, celle-ci étant fonction avant tout de l'alimentation éventuelle des zones fruitières par substitution aux centrales Diesel existantes.

Au premier stade du développement, un débit minimum constant de $4 \mathrm{~m}^{3} / \mathrm{s}$ peut être utilisé sans aucun prélèvement sur les eaux du lac autre que les fuites naturelles qui forment une partie de ce débit.

Un barrage de prise de faible hauteur, une amenée de $800 \mathrm{~m}$ de long, partie en conduite, partie en canal à l'air libre et une conduite forcée de $400 \mathrm{~m}$ environ suffiraient pour alimenter deux groupes de $3.750 \mathrm{kVA}$, la hauteur de chute utilisée étant limitée à $150 \mathrm{~m}$.

Entre ce premier stade et celui correspondant au maximum productible, il est aisé, sans aucun gaspillage d'énergie, de prévoir un développement progressif de cet ensemble, dont les ressources sont relativement énormes. En effet, après étanchement des résurgences du rio Zacapa et obturation, par un petit barrage, de l'émissaire sud (rio Jaitique), le débit moyen prélevé sur le lac pourra atteindre $22 \mathrm{~m}^{3} / \mathrm{s}$. Ce débit pourrait même être porté à $30 \mathrm{~m}^{3} / \mathrm{s}$ par des travaux de dérivations d'eaux provenant d'autres bassins versants.

Ia hauteur totale de chute utilisable étant de $540 \mathrm{~m}$ environ, c'est une productibilité annuelle de l'ordre de 1.000 millions de kWh qui peut être attendue de l'aménagement au stade définitif, correspondant à une puissance continue de $120.000 \mathrm{~kW}$.

I.e projet réunit les avantages suivants :

- Débit relativement élevé et peu variable en raison d'une alimentation en partie souterraine;

- Grand réservoir naturel à l'amont (lac Yojoa) permettant les prélèvements en période sèche;

- Hauteur de chute utilisable importante;

-. Possibilités très grandes d'extension progressive de la puissance;

- Rentabilité accrue par les irrigations nouvelles que permettrait un programme d'utilisation combinant les besoins électriques et agricoles;

- Et surtout, position centrale permettant immédiatement la substitution à l'énergie thermique des centrales diesel de la zone côtière nord actuellement la plus développée, d'énergie d'origine hydraulique, en même temps que le développement de la région de Tegucigalpa et de l'axe nordsud du pays.

II s'agira d'ure énergie d'accumulation pour sa plus grande part, et la proximité relative des futures usines du rio Lempa au Salvador devra inciter à prévoir une interconnexion future des deux ensembles. Il serait ainsi possible, dans un avenir certes encore lointain, de réaliser d'intéressants échanges d'énergie entre les usines d'accumulation du rio Lindo et celles du rio Lempa. les projets de ces dernières devront réserver, pour le cas où cette hypothèse viendrait à se réaliser, des possibilités d'augmentations futures de puissance permettant d'utiliser des débits nota. blement plus importants que ceux qui seraient autrement aménagés. Ceux-ci, insuffisamment régularisés par des réservoirs de barrage dont la capacité décroîtra d'ailleurs avec le temps, ne pourraient guère être très notablement supérieurs aux débits d'ćtiage. Durant la saison sèche, le lac Yojoa fournirait l'énergie de pointc au Salvador, tandis que durant les mois de hautes eaux, les usines du Lempa alimenteraient le réseau du Honduras, ce qui équivaudrait à une mise en réserve d'une partie de l'énergie sauvage de cette rivière. 


\section{Nicaragua}

La quasi-totalité de l'énergie électrique produite actuellement dans le pays est d'origine thermique. La région de la capitale, Managua, est la seule à bénéficier d'une puissance installée notable. Celle-ci, qui est actuellement de $10.000 \mathrm{~kW}$, semble devoir devenir rapidement insuffisante, le taux annuel d'accroissement de la demande d'énergie ayant été de $15 \%$ au cours des dernières années.

La nécessité d'importer le combustible a poussé récemment les pouvoirs publics à rechercher si des ressources hydrauliques pourraient être mises en valeur.

Depuis 1952, des études hydrologiques ont été entreprises avec l'aide technique des U.S.A. (au titre du point IV), notamment sur le rio Tuma.

D'autres possibilités existent qui utiliseraient les ressources potentielles offertes par les grands lacs Managua et Nicaragua. Leur mise en valeur nécessiterait toutefois ds travaux importants et devrait faire l'objet, d'ores et déjà, d'études suivies.

\section{Le rio Tuma.}

Cette riviere est un aflluent du rio Grande de Matagalpa, tributaire de l'Atlantique (fig. 11), Les premières reconnaissances sont récentes (1951) et les mesures hydrologiques ne portent encore que sur un temps encore très limité.

Cependant, on peut déjà affirmer qu'en dépit de la distance élevée $(160 \mathrm{~km})$ de cette rivière aux centres de consommalion, c'est elle qui apportera dans les meilleures conditions la solution recherchée.

Elle coule, en effet, dans son cours supérieur, en amont de son confluent avec le rio Yasica dans des gorges rocheuses étroites el profondes où l'on pourrait trouver plusieurs emplacements favorables à la construction d'un barrage-vonte de $40 \mathrm{~m}$ à $60 \mathrm{~m}$ de hauteur.

Il semble résulter des premières années de jaugeages qu'un débit moyen de $10 \mathrm{~m}^{3} / \mathrm{s}$ pourrait être utilisé après régularisation, à condition qu'une retenue de 170 millions de $1 \mathrm{~m}^{3}$ puisse ctre créée. La hauteur totale de chute utilisable, qui devrait être fractionnée en deux ou trois

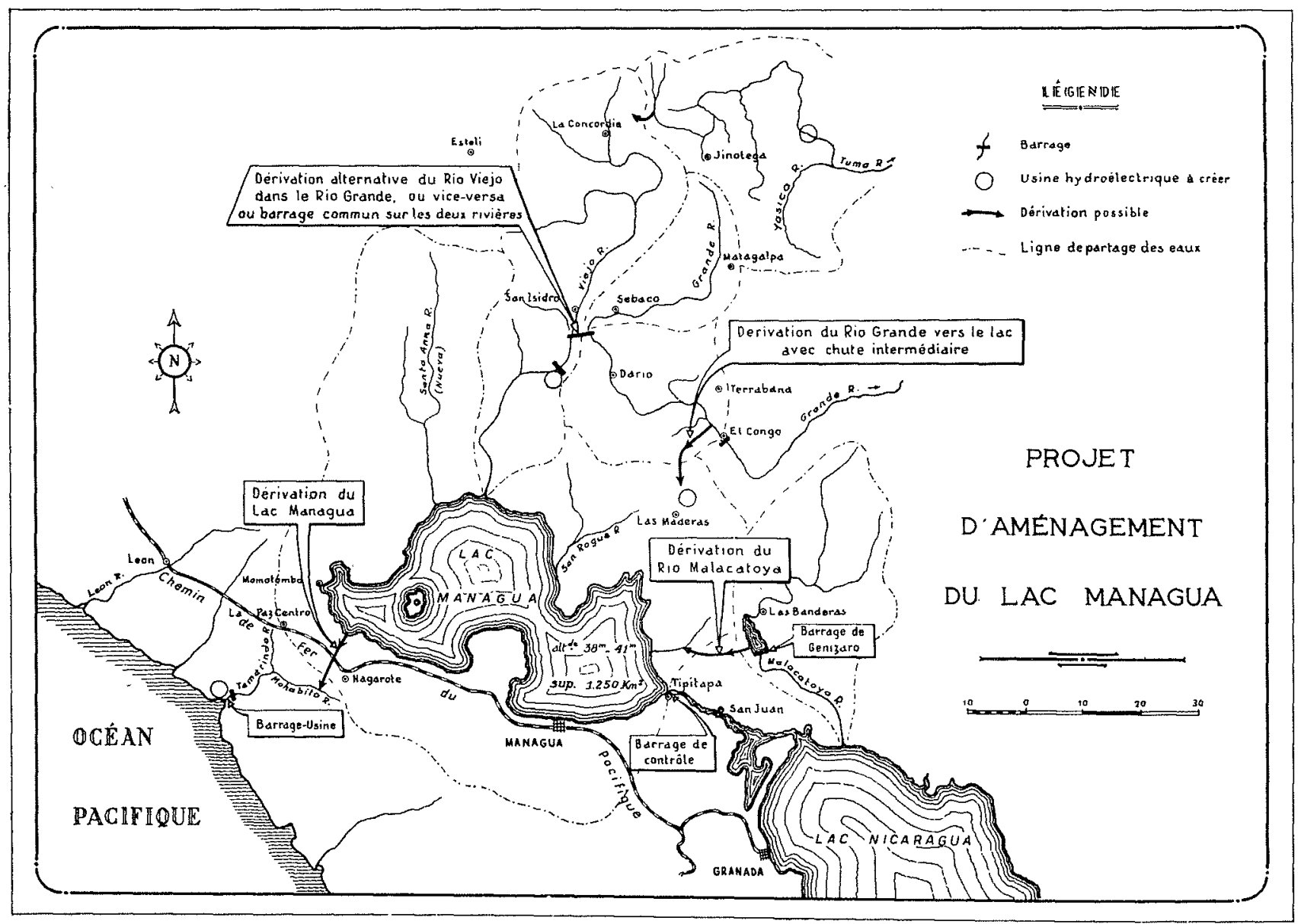

FIG. 11

Lac Managua et rio Tuma (carte). 
installations, serait au total de $550 \mathrm{~m}$ environ.

L'ensemble pourrait assurer une productibilité annuelle de $370 \mathrm{GWh}$, et justifier ure puissance installée de $100.000 \mathrm{~kW}$ environ. Il s'agit là d'eslimations prudentes, qui pourraient se révéler au-dessous de la réalité.

\section{Le lac Managua.}

Le lac Managua, dont la superficie propre est d'environ $1.250 \mathrm{~km}^{2}$, est alimenté par un bassin versant de $3.950 \mathrm{~km}^{2}$, soit $5.200 \mathrm{~km}^{2}$ au total. Il est séparé de la côte par un isthme qui se rétrécit au droit du rio Tamarindo jusqu'à $25 \mathrm{~km}$ de largeur, et s'abaisse en même temps juscju'à $20 \mathrm{~m}$ seulement au-dessus du niveau moyen du lac, lequel est d'cnviron $39 \mathrm{~m}$ audessus du niveau de la mer.

Le principal tributaire du lac, le rio Viejo, nait tout près des sources mêmes du rio Tuma, dans des montagnes qui ne s'élèvent guère au-dessus de $1.500 \mathrm{~m}$. Entre la ville de Sébaco (fig. 11) et le lac, cette rivière présente un troncon de $16 \mathrm{~km}$ environ de 0,02 de pente moyenne oì une chute pourrait être aménagée (Santa Barbará).

Peu en aval de Sébaco, qui est située sur le rio Grande, aflluent de l'Atlantique, les eaux de cette rivière et celles du rio riejo inondent en périodes de crues les plaines envirommantes, si bien que leurs bassins versants sont alors pratiquement confondus $\left(1.780 \mathrm{~km}^{2}\right.$ au total).

Le seul exutoire superficiel du lac est le rio Tipitapa qui est normalement à sec et qui déverse dans le lac Nicaragua trois ou quatre fois par siècle seulement. Il existe entre ce lac et le lac Managua, qui est plus élevé, une dénivellation variable de l'ordre de $8 \mathrm{~m}$ dont près de $5 \mathrm{~m}$ à l'origine de l'exutoire, près du village de Tipitapa.

Des divergences existent au sujet des pertes qui, selon les uns, alimenteraient le lac Nicaragua par des courants souterrains aboutissant dans la zone aval du rio Tipitapa, selon les autres amèneraient les eaux de fuites vers le Pacifique. Diverses études ont été faites, comportant mesures de salinité, de conductivité électrolytique, et $\mathrm{du} \mathrm{pH}$, sur des prélèvements faits en divers points, les eaux du lac Managua étant saumâtres, mais sans conclusion formelle. Il semble toutefois que l'influence des fuiles, si celles-ci existent, soit inférieure à celle des pertes par évaporation.

Ceci dit, on voit que les projets d'utilisation du lac Managua devraient amener au lac des quantités d'eau nouvelles représentant non seulement les débits turbinés, mais encore ceux correspondant aux évaporations et aux fuites qui à eux seuls sont de l'ordre de $4 \mathrm{~m}^{3} / \mathrm{s}$.

Supposant ce problème résolu (nous verrons plus loin comment il pourrait l'être), les eaux seraient prélevées sur le lac par un canal en tranchée de $13 \mathrm{~km}$ de long à travers l'isthme côtier et amenées dans le lit du rio Tamarindo par l'intermédiaire d'un affluent. Un barrage serait construit sur cette rivière près de son embouchure, créant une chute dont la hateur nette ne dépasserait guère $20 \mathrm{~m}$.

Il s'agirait de travaux relativement très importants qui ne pourraient être justifiés que par la possibilité de résoudre aisément le problème relatif aux apports d'eaux supplémentaires.

Plusieurs solutions ont été envisagées, faisant intervenir, soit des dérivations de rivières avec utilisation des chutes intermédiaires, soit le prélévement des débits nécessaires par pompage dans le lac Nicaragua.

Le croquis (fig. 11) indique les dérivations qui ont été proposées.

La dérivation du rio Grande en aval de Sébaco dans le rio Viejo permettrait en outre, par la construction d'un long barrage en lerre, la régularisation des débits qui, avant de parvenir au lac, seraient utilisés, comme nous l'avons vu plus haut, dans un aménagement à réaliser en aval. Un barrage d'une trentaine de mètres de hauteur permettrait d'utiliser une chute de près de $100 \mathrm{~m}$.

D'après de premières investigations, un débit régularisé de lordre de $15 \mathrm{~m}^{3} / \mathrm{s}$ pourrait être obtenu. Si les études ullérieures confirmaient ces premières donnćes, ce serait peut-être le còté le plus intéressant de cette dérivation, encore que le coût du barrage de régularisation semble devoir être relativement très élevé.

La dérivation du rio Grande au lieu dit El Congo est mentionnée en variante par ccrtains projeteurs, avec possibilité d'utiliser une chute de $90 \mathrm{~m}$, dans des conditions qui, à vrai dire, paraissent économiquement assez peu satisfaisantes.

Enfin, la dérivation par un barrage qui serail construit en aval de Las Banderas sur le rio Malacatoya, tributaire du lac Nicaragua, aurait pour inconvénient majeur de priver d'irrigations la zone nord-ouest de ce lac.

Nous croyons d'ailleurs que les quantités d'eaux dérivables en vue d'une production hydroélectrique ne justifieront jamais les importants investissements qu'elles supposent.

Le prélèvoment d'eaux par pompage dans le lac Nicaragua serait-il plus rentable?

Si l'on ajoute à la hauteur effective de refoulement de $7,50 \mathrm{~m}$ une hauteur représentative des pertes (rendement de l'usine de pompage, pertes électriques, perte de charge), on arrive à la conclusion que la chute nette résiduelle rćellement utilisable serait de l'ordre de $9 \mathrm{~m}$.

Le débit nécessaire pour obtenir, avec une chute aussi réduite, une puissance correspondant à celle qui sera demandée au moment où l'aménagement serait achevé, et qui peut être évaluce à $20.000 \mathrm{~kW}$ de puissance continue, serait de l'ordre de $220 \mathrm{~m}^{3} / \mathrm{s}$.

Un prélèvement de cette importance nécessite- 
rait tout d'abord que soit obluré par un barrage l'émissaire du lac Nicaragua, le rio San Juan.

Ainsi donc, quelle que soit la solntion adoptée, on voit que le canal d'amenée qui nécessiterait une tranchée de $13 \mathrm{~km}$ de long et de plus de $20 \mathrm{~m}$ de profondeur maximum, ainsi que les autres importants ouvrages du projet, devraient encore être complétés par un ensemble nécessitant des travaux considérables qui paraissent hors de proportion avec les quantités d'énergie disponibles.

Nous avons cependant exposé les grandes lignes de ces projets, dont on reparle de temps à autre, bien qu'ils soient, à notre avis, sans intérêt pratique, en raison notamment de la possibilité d'utiliser directement les eaux du lac Nicaragua dans des conditions paraissant a priori plus favorables.

\section{Le lac Nicaragua.}

Le lac Nicaragua, qui a une superficie propre de $7.700 \mathrm{~km}^{2}$, draine un bassin versant de $34.000 \mathrm{~km}^{2}$ (y compris celui du lac Managua), bassin dont le point culminant attcint $1.800 \mathrm{~m}$ dans les montagnes du Costa Rica, qui bordent ses rivages sud-est.

L'émissaire du lac est le rio San Juan, long de $200 \mathrm{~km}$ environ, tributaire de la mer des Caraïbes. Le niveau moyen du lac oscille entre 32 et $33,50 \mathrm{~m}$.

Il existe au sujet de ce lac et de sa région des données hydrologiques, géologiques et topographiques relativement complètes et précises, grâce aux études faites entre 1826 et 1901, en 1931 et en 1941 par le Corps of Engineers des Etats-Unis en. vue de la création d'un canal transocéanique dont le tracé devait suivre le rio San Juan, emprunter le lac et franchir l'isthme dans sa partie la plus basse qui est aussi la plus étroite (fig. 12).

Les études hydrologiques portant sur un cycle de 14 ans concluent à un débit moyen du rio San Juan, au lieu dit Conchuda, de $600 \mathrm{~m}^{3} / \mathrm{s}$, la superficie du lac étant suffisante pour une régularisation interannuelle, sans modifier sensiblement le marnage naturel.

Plusieurs projets d'aménagement ont été envisagés. Dans leur phase de développement maximum, ils comprendraient un barrage sur le rio San Juan, où deux emplacements possibles ont été retenus, l'un à Conchuda, l'autre au confluent du rio Sábalos.

Le premier emplacement, à l'amont immédiat du confluent avec le rio San Carlos, nécessiterait un barrage déversoir en béton de $600 \mathrm{~m}$ de long et de $45 \mathrm{~m}$ de hauteur maximum. Les études du Corps of Engineers auraient conclu à des conditions géologiques satisfaisantes. La chute brute utilisable serait de $12 \mathrm{~m}$ entre les cotes 32,50 et 20,00 .

Une variante de ce projet, peut-être plus rationnelle en raison du raccourcissement de la distance aux lieux de consommation, et d'une meilleure utilisation résultant de l'augmentation de la chute, consisterait à détourner les eaux du lac vers le I'acifique par l'intermédiaire d'un canal d'amenée traversant l'isthme côtier. Ce canal, capable d'un débit de $600 \mathrm{~m}^{3} / \mathrm{s}$, aurait une Iongueur d'environ $12 \mathrm{~km}$, et nécessiterait le percement d'une tranchée de $24 \mathrm{~m}$ de profondeur maximum à travers la ligne de partage des eaux. Il suivrait, au départ du lac, le tracé projeté du grand canal transocéanique, en remontant, au départ du lac, le rio Las Lajas. Il amènerait les eaux prélevées vers le rio Grande où deux emplacements de barrages-usines paraissent possibles d'après les données topographiques existantes. L'un d'eux, situé à l'amont immédiat de Brito, nécessiterait un barrage d'environ $1.800 \mathrm{~m}$ de longueur en crête et $30 \mathrm{~m}$ de hauteur au-dessus du lit, créant une chute brute de $24 \mathrm{~m}$. On pourrait attendre de cet ensemble une productibilité annuelle de l'ordre de 1.000 millions de $\mathrm{kWh}$. La puissance installée pourrait donc atteindre $180.000 \mathrm{~kW}$.

Le barrage du rio San Juan pourrait, ou bien ètre maintenu au lieu dit Conchuda, ce qui laisserait la possibilité de produire dans cette région une partie de l'énergie électrique disponible qui pourrait être dirigée soit vers San José de Costa Rica, soit vers la côte Caraïbe, soit de construire le barrage de contrôle du rio San Juan à l'aval immédiat du confluent du rio Sábalos. Une hauteur de barrage de 12 à $15 \mathrm{~m}$ serait alors suffisante, mais la longueur de l'ouvrage serait encore de $600 \mathrm{~m}$, et le bassin versant total étant moindre que pour un barrage situé à Conchuda, l'énergie productible totale s'en trouverait réduite de $30 \%$.

Le projet d'aménagement du lac Nicaragua en vue de la production d'énergie électrique mérite sans aucun doute une ample considération.

Cependant, une des difficultés sera peut-être d'ordre politique. Le rio San Juan est en effet dans son cours inférieur un fleuve frontière entre deux Etats et les nécessités de la navigation devront sans doute entrer en ligne de compte. L'intérêt des deux pays voisins les incitera vraisemblablement dans l'avenir à rechercher un accord profitable à chacun d'eux, qui leur permettra de parfaire leur développement économique.

La mise à exécution, dont on reparle plus que jamais, du projet de canal transocéanique, viendrait sans aucun doute apporter à ce projet quelques importantes modifications. 


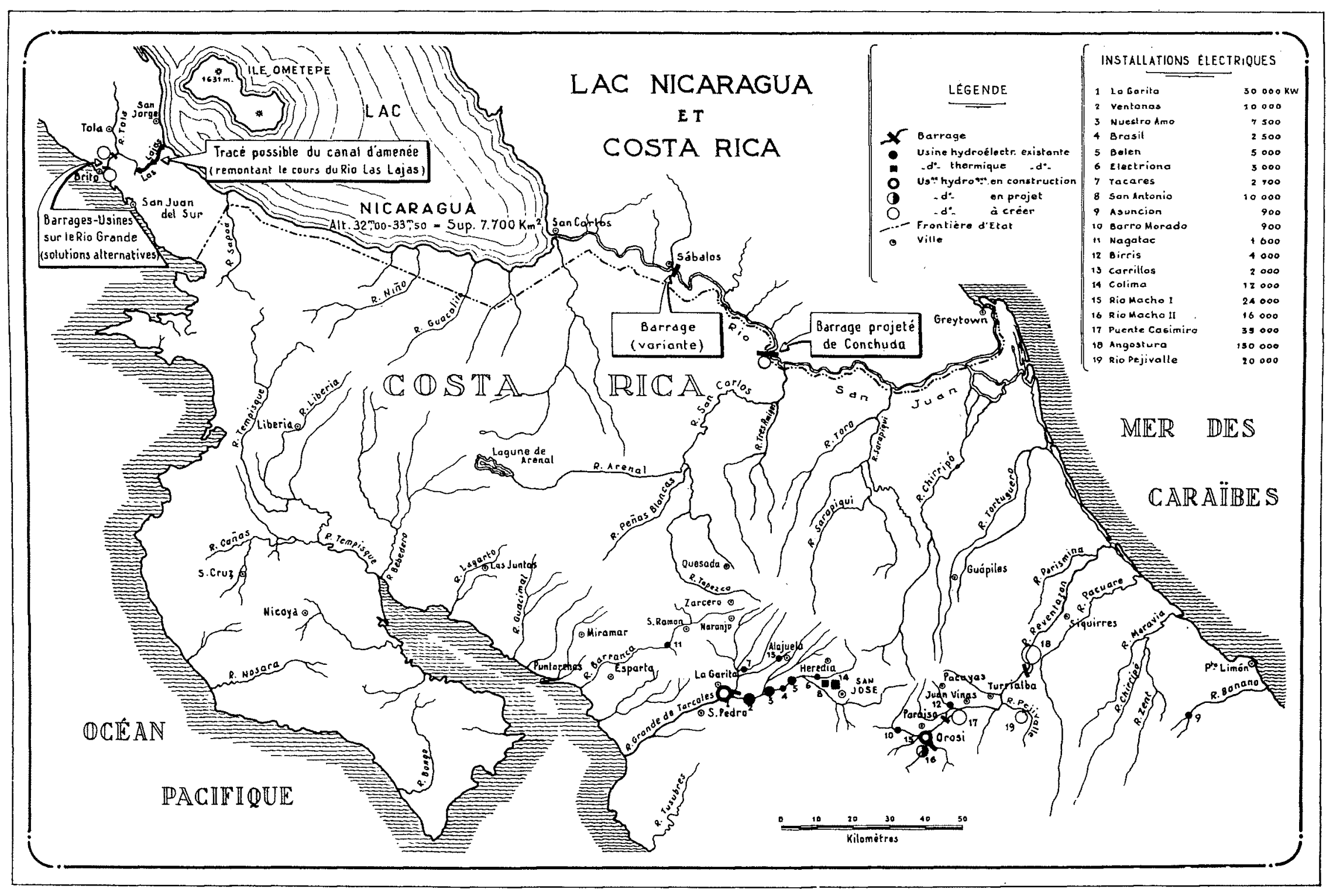




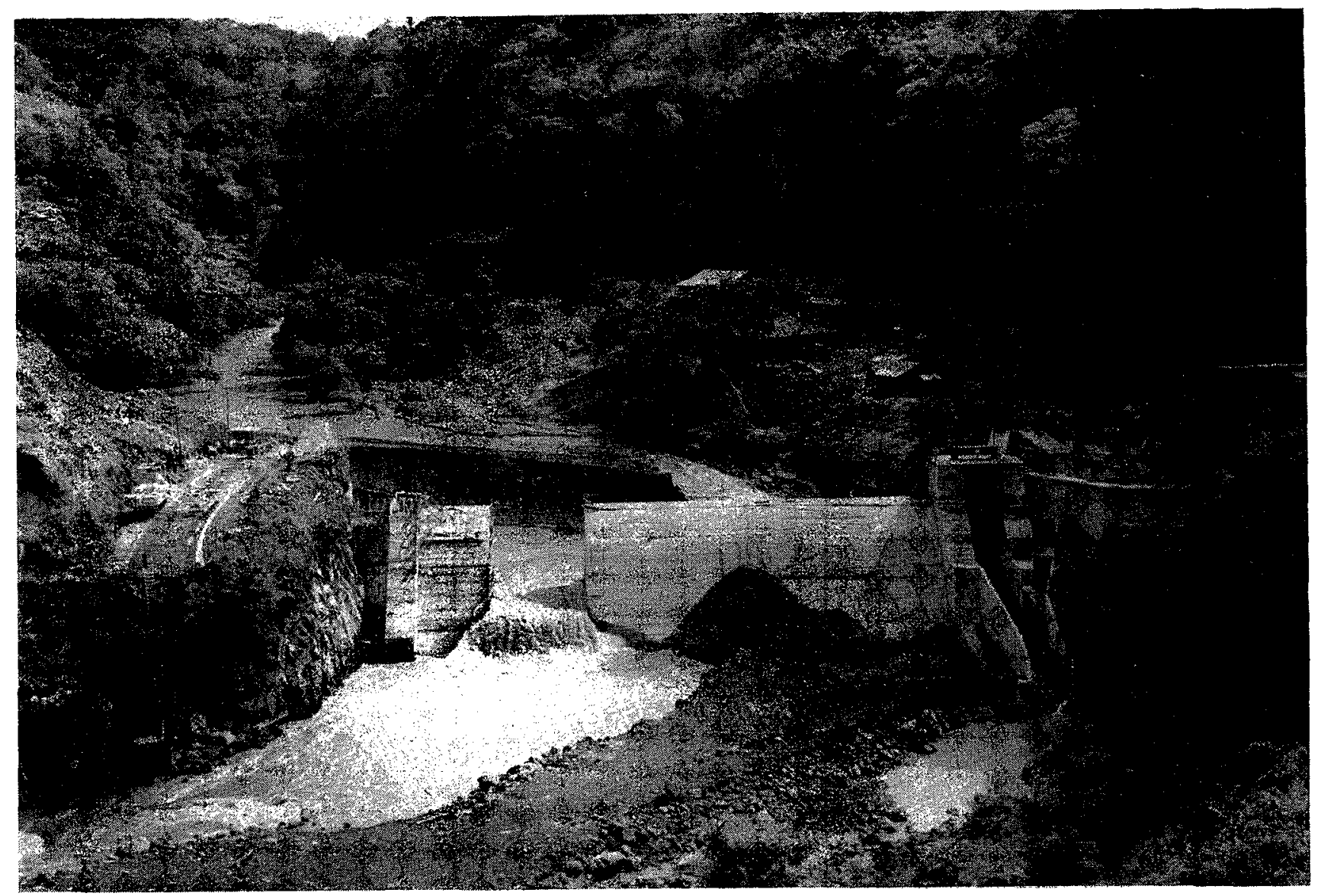

FIG. 13

Travaux d'aménagement hydroèlectrique de La Garita. - Barrage de prise.

\section{Costa Rica}

Le Costa Rica est le pays d'Américue centrale où la consommation d'énergie électrique est la plus élevée par rapport au nombre d'habitants. Elle approche en effet de $500 \mathrm{kWh}$ annuels par habitant pour la zone centrale du pays qui renferme plus de $50 \%$ de la population.

Ia puissance inștallée dépassera prochainement, lorsque l'aménagement hydro-électrique sommairement décrit plus bas de La Garita aura été mis en service, le chiffre de $85.000 \mathrm{~kW}$. Sur ce total, les usines hydro-électriques comptent pour $70.000 \mathrm{~kW}$. Nous ne décrirons pas les aménagements hyzlro-électriques existants, dont certains atteignent cependant une puissance de $10.000 \mathrm{~kW}$ (fig. 12).

La demande d'énergie s'accroît à une cadence rapide - elle double en huit ans - et la mise en service récente d'une centrale diesel de $10.000 \mathrm{~kW}$ n'a apporté qu'une amélioration temporaire.

L'Instituto Costarricense de Electricidad (I.C.E.) qui a la charge de l'électrification du pays a étudié un programme important d'aménage- ments hydro-électriques dont il poursuit sans désemparer la réalisation méthodique.

Le premier d'entre eux, en cours d'exécution, est celui de La Garita, sur le rio Grande de Tarcoles. Les études actuelles pour la mise en valeur d'autres chutes se poursuivent notamment sur le rio Reventazón et ses affluents supérieurs.

\section{L'aménagement hydro-électrique de La Garita.}

Les travaux de La Garita ont été commencés en 1954. L'usine hydro-électrique est située sur le cours supérieur du rio Grande, tributaire du Pacifique, et utilise les eanx correspondant à un bassin versant de $644 \mathrm{~km}^{2}$.

Les données hydrologiques ne portent que sur un temps relativement court. Le débit minimum d'étiage n'a jamais été inférieur à $15 \mathrm{~m}^{3} / \mathrm{s}$ correspondant au mois le plus sec de la période sèche de décembre à avril durant laquelle les précipitations sont nulles. De mai à novembre, la hauteur mensuelle moyenne de pluie est de $369 \mathrm{~mm}$ dans la zone haute du bassin versant, soit $2.600 \mathrm{~mm}$ par an.

Les ouvrages sont prévus pour l'utilisation d'un 

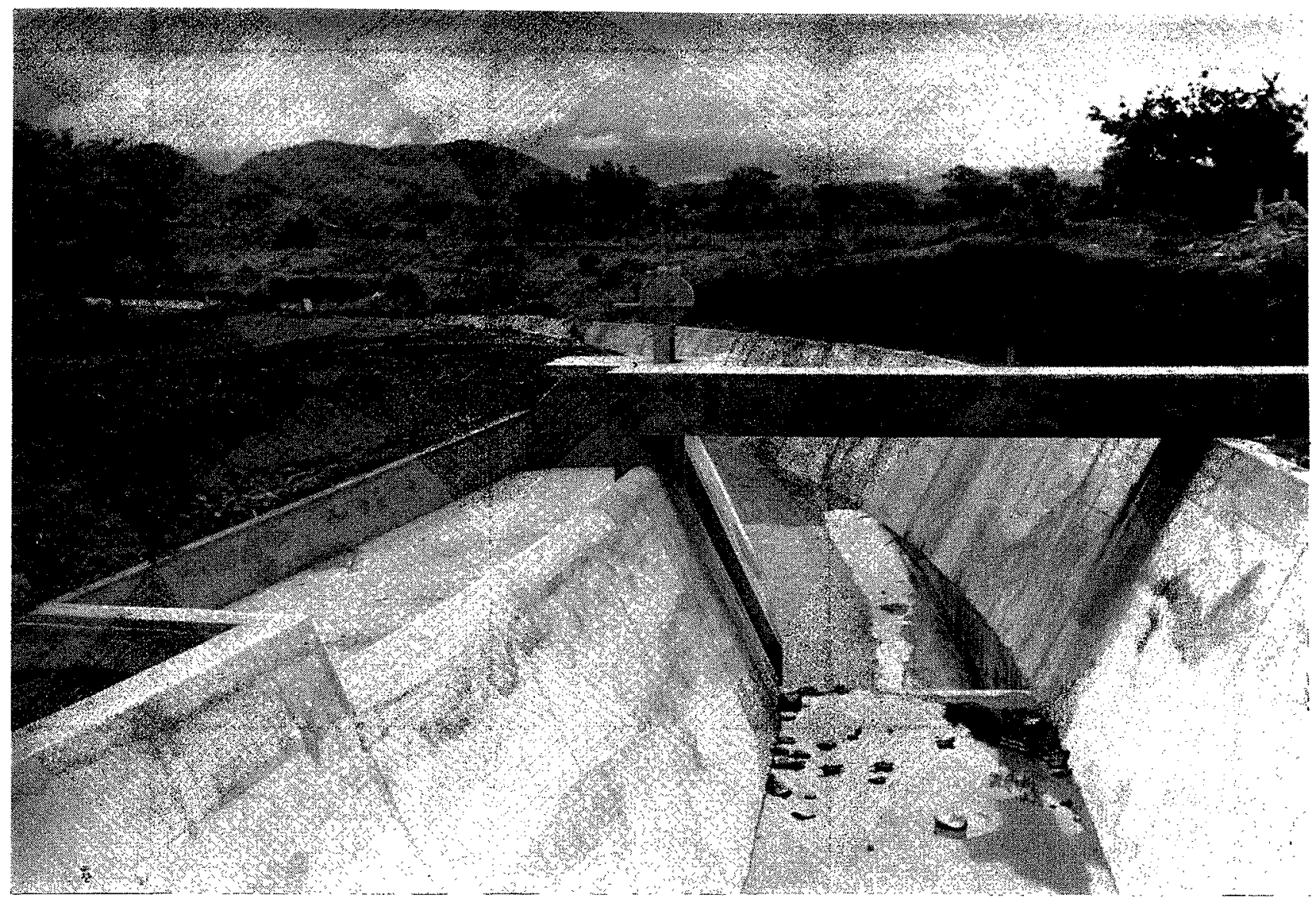

FIG. 14

Travaux d'aménagement hydroélectrique de La Garita. - Canal d'amenée.

déhit dérivé de $17 \mathrm{~m}: \mathrm{s}$, la prise d'eau est constituée par un barrage-poids de $18 \mathrm{~m}$ de hauteur maximum, comportant un déversoir de $59 \mathrm{~m}$ de longueur prévu pour une crue maximum de $1.700 \mathrm{~m}^{3} / \mathrm{s}$, et une vanne de fond de $4 \mathrm{~m} \times 5 \mathrm{~m}$ (fig. 13) (1).

La conduite d'amenée, de $6.200 \mathrm{~m}$ de longueur, comprend trois sections avec revêtement bétonné, de $4.396 \mathrm{~m}$ de longueur totale en galerie en forme de fer à cheval de $3,35 \mathrm{~m}$ de diametre, trois sections de canal à l'air libre sur $1.563 \mathrm{~m}$ (fig. 14), deux traversées d'affluents, l'une en pont-canal de $23 \mathrm{~m}$ de long, l'autre en siphon de $244 \mathrm{~m}$ de long et de 3,24 m de diamètre.

Sur le parcours de la conduite d'amenée est prévu un dessableur à l'air libre entre deux troncons de tunnel. A la traversée, par les ouvrages d'amenée, d'une vallée secondaire, a été aménagée, à l'aide d'un barrage en terre de $270 \mathrm{~m}$ de long et de $12 \mathrm{~m}$ de hauteur maximum, une réserve

(1) Les photographies (fig. 13,14 et 15 ), sont dues à l'obligeance de l'ingénieur J.-M. Dexco, directeur de l'Instituto Costarricense de Electricidad, organisnic national autonome. d'utilisation journaliere de $430.000 \mathrm{~m}:$ utilisables permettant des pointes de débit de $26 \mathrm{~m}^{3} / \mathrm{s}$.

La conduile forcée, dont le diamètre varie de $2,80 \mathrm{~m}$ à $3 \mathrm{~m}$, a une longueur totale de $456 \mathrm{~m}$ pour une chute brute variant de 153 à $160 \mathrm{~m}$ (fig. 15).

L'usine comporte deux turbines Francis à axe vertical de $23.600 \mathrm{ch}$ sous $151,50 \mathrm{~m}$ de chute nette et deux alternateurs de $15.000 \mathrm{~kW}$, tournant à $514 \mathrm{tr} / \mathrm{mn}$.

Elle a été prévue, ainsi que la chambre de mise en charge, de manière à permettre l'installation de deux groupes supplémentaires de $15.000 \mathrm{~kW}$ qui seraient ultérieurement alimentés par les eaux dérivées du rio Virilla, aflluent important, dont le confluent avec le rio Grande est à $800 \mathrm{~m}$ à l'aval de l'usine de La Garita.

La mise en service de la premiere tranche est prévue pour 1957.

\section{Les projets d'aménagement du rio Reventazón.}

Le rio Reventazón, tributaire de la mer des Caraibes, se forme sur le versant nord-est de la Cordillère de Talamanca où son bassin versant atteint une altitude de pris de $3.500 \mathrm{~m}$. Il cons- 


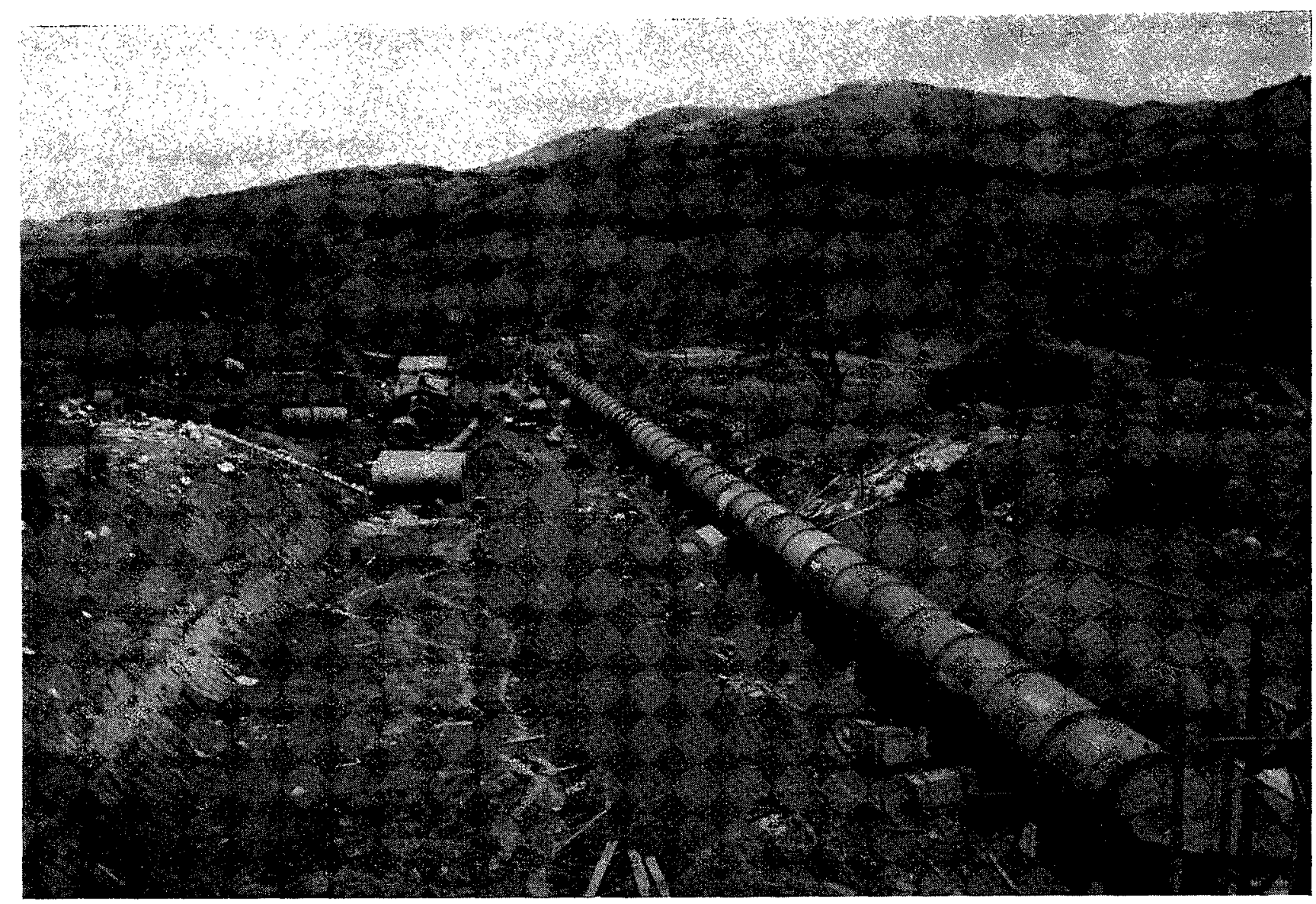

Fig. 15

Travaux d'aménagement hydroélectrique de La Garita. Conduite forcée (trinvax en cours).

titue, avec ses affluents supérieurs, l'une des sources d'énergie les plus importantes du pays. La mise en valeur de ce potentiel comprendrait les aménagementś ci-après :

- Sur le rio Macho, dont le confluent avec le rio Reventazon est à une cote voisine de $1.090 \mathrm{~m}$, une chute de $900 \mathrm{~m}$ de hauteur totale.

Celle-ci serait divisée en deux tronçons aménagés séparément. L'étude correspondant au premier tronçon est en cours. Le projet prévoit l'installation de deux groupes de $12.000 \mathrm{~kW}$ dont le premier serait mis en service en février 1959 , le deuxième en 1961.

En tête du deuxième tronçon, qui justifierait une puissance installée de $16.000 \mathrm{~kW}$, sera créée une réserve de régularisation de 10 millions de $\mathrm{m}^{3}$;

-- Sur le rio Reventazón lui-même :

La chute de Puente Casimiro, qui comprendrait un barrage de prise en béton avec couronnement à la cote 986 . Une galerie d'amenée d'environ
$8 \mathrm{~km}$ conduirait les eaux jusqu'au confluent avec le rio Birris, à la cote 800 où serait construite l'usine avec une puissance installée de $35.000 \mathrm{~kW}$ pour un débit minimum moyen de $15 \mathrm{~m}^{3} / \mathrm{s}$.

La chute d'Angostura située à l'aval du rio Pejivalle, à $36 \mathrm{~km}$ environ à l'aval du harrage de Puente Casimiro, qui nécessiterait la construction d'un barrage de $50 \mathrm{~m}$ de hauteur environ au-dessus du niveau du lit et de $350 \mathrm{~m}$ environ de longueur en crête fondé dans un massif andésitique. Les eaux seraient prises à la cote 580 par une galerie d'amenée percée sur la rive droite. Deux solutions sont à étudier, suivant la position de l'usine à la cote 430 ou, plus à l'aval, à la cote 350 environ. Dans la première solution, la galerie d'amenée aurait une longueur de $4 \mathrm{~km}$, percée presque entièrement dans la brèche sédimentaire. et son exécution ne présenterait pas de difficultés particulières. La conduite forcée serait courte et fondée sur un terrain solide. Au contraire, la deuxième solution exigerait une galerie de $9 \mathrm{~km}$ à travers des terrains peut-être plus difficiles, dont l'étude géologique 
n'est d'ailleurs pas encore achevée, el l'établissement d'une conduite forcée de plus de $2.000 \mathrm{~m}$.

Le débit minimum moven disponible à Angostura serait de $42 \mathrm{~m}^{3} / \mathrm{s}$.

La puissance installée pourrait être ainsi de l'ordre de $100.000 \mathrm{~kW}$ ou de $150.000 \mathrm{~kW}$ suivant la solution choisic, en admetlant un coefficient d'utilisaticn de 50 g.

Les conditions topographiques semblent devoir permettre d'améliorer encore ces chiffres par la création d'un réservoir de régularisation. Il reste à comparer financièrement les avantages de cette régularisation, conjute tenu de la durée probable de la réserve utile créée. Celle-ci dépend strictement du débit solide de la rivière qui est relativement élevé. Cet aspect n'a pas échappé aux techniciens de l'I.C.E. qui l'étudient avec un soin tout particulier;

- Enfin, d'autres aménagements pourraient être étudiés soit sur d'autres troncons du rio Reventazón, soit sur ses afluents supérieurs.

Sur le rio Pejivalle en particulier, affluent de la rive droite en anont de la retenue projetée d'Angostura, une puissance de $20.000 \mathrm{~kW}$ pourrait être installée.

On admet que l'ensemble du bassin supérieur du rio Reventazón jusqu'au rio Pejivalle inclus pourrait justifier une puissance installée totale de l'ordre de $140.000 \mathrm{~kW}$.

\section{Autres ressources hydro-électriques.}

Il existe encore dans ce pays d'autres ressources hydro-électriques potentielles importantes; nous avons noté seulement celles qui paraissent devoir être étudiées dans un avenir rapproché.

Si le projet du lac Nicaragua venait à être réalisé, une partie de l'énergie produite devrait, semble-t-il, revenir au Costa Rica qui détient une partie non négligeable du bassin versant du lac, surtout si le barrage sur le rio San Juan étail construit à Conchuda. La distance d'amenće de l'energie qui pourrait êlre produite à Conchuda jusqu'aux centres de consommation du pays ne serait d'ailleurs pas probibitive $(90 \mathrm{~km})$.

$$
\text { 济 }
$$

En conclusion, on peut observer que si l'Aniérique centrale ne semble pas avoir ćté favorisće par des ressources locales en combustibles, elle se trouve au contraire relativement bien pourvue en ce qui concerne ses ressouces énergétiques hydrauliques. La mise en valeur de ces dernicres, qui déjà dans plusiəurs pays de l'isthme centro-américain se poursuil d'une manière progressive et rationnelle, viendra apporter à brève échéance à l'économie de cette région un essor nouveau et augmenter encore le bien-être de ses populations.

C'est, en terminant, le veu que nous formulerons à l'adresse de ces pays si attachants à de nombreux titres.

\section{ACHETEUR}

TURBINE MODERNE

175 à 225 ch environ - Chute $3,60 \mathrm{~m}$

ALTERNATEUR

100 à $120 \mathrm{kVA}-220$ volts -50 périodes

Ecrire HOUILLE BLANCHE, $n^{\circ} 201$ 